\title{
A BASIS OF BIDETERMINANTS FOR THE COORDINATE RING OF THE ORTHOGONAL GROUP
}

\author{
GERALD CLIFF
}

\begin{abstract}
We give a basis of bideterminants for the coordinate ring $K[O(n)]$ of the orthogonal group $O(n, K)$, where $K$ is an infinite field of characteristic not 2. The bideterminants are indexed by pairs of Young tableaux which are $O(n)$-standard in the sense of King-Welsh. We also give an explicit filtration of $K[O(n)]$ as an $O(n, K)$-bimodule, whose factors are isormorphic to the tensor product of orthogonal analogues of left and right Schur modules.
\end{abstract}

\section{INTRODUCTION}

Let $K$ be an infinite field. It was shown by Mead $[\mathrm{M}]$ and by Doubilet-Rota-Stein [DRS] that the polynomial ring $K[X(i, j)]$ in $n^{2}$ variables has a basis of bideterminants, indexed by pairs of standard Young tableaux. DeConcini-Eisenbud-Procesi [DEP] used these bideterminants to give an explicit filtration of $K[X(i, j)]$, as a $G L(n, K)$-bimodule, whose factors are isomorphic to $L_{\lambda} \otimes_{\lambda} L$ where $L_{\lambda}$ and ${ }_{\lambda} L$, are left and right Schur modules, respectively, corresponding to partitions $\lambda$ into at most $n$ parts. At characteristic $0, L_{\lambda}$ and ${ }_{\lambda} L$ are irreducible polynomial representations of the general linear group $G L(n, K)$.

We wish to investigate the situation where $G L(n, K)$ is replaced by the orthogonal group $O(n, K)$. We assume that the characteristic of $K$ is not 2. The bideterminants which are a basis of $K[X(i, j)]$ are not linearly independent as functions on $O(n, K)$. We show that the coordinate ring $K[O(n)]$ of $O(n, K)$ has a basis of bideterminants $[S: T]$ where $S$ and $T$ are $O(n)$-standard in the sense of King-Welsh $[\mathrm{KW}]$. As far as we are aware, this is the first known explicit basis of $K[O(n)]$. We also find an explicit $O(n, K)$-bimodule filtration of $K[O(n)]$ with factors isomorphic to $L_{O}^{\lambda} \otimes{ }^{\lambda} L_{O}$, where $L_{O}^{\lambda}$ and ${ }^{\lambda} L_{O}$, are analogues of left and right Schur modules, for the orthogonal group.

Our first problem is to show that the $O(n)$-standard bideterminants generate $K[O(n)]$; to do this we give a straightening algorithm. We then give our filtration of $K[O(n)]$, from which linear independence of the $O(n)$-standard bideterminants can be deduced.

We first prove these results under the assumption that the field $K$ has characteristic 0 . We then show that there are analogues of these results where $K$ is replaced by the ring $\mathbb{Z}[1 / 2]$, and then by base change, for fields of odd characteristic.

In $[\mathrm{KW}]$ a straightening method is given for an $O(n)$-module (assuming $K=\mathbb{C}$ ) denoted $O^{\lambda}$ which is a factor module of a submodule of $V^{\otimes m}$ where $V$ is the natural $O(n)$-module. (This is defined in Section 6 below, where it is shown that $O^{\lambda}$ is isomorphic to $L_{O}^{\lambda}$.) Straightening in $[\mathrm{KW}]$ is done using a modification of Berele's method [B] (see also [Don]) for the symplectic group, using a suitable quotient of the tensor algebra. Our straightening method is more complicated than that of $[\mathrm{KW}]$, as we need to use tableaux of different shapes and

This research was supported in part by a grant from the Natural Sciences and Engineering Research Council of Canada. 
sizes. Linear independence of the basis in $[\mathrm{KW}]$ for $O^{\lambda}$ is deduced from work of Proctor [Pro].

We also give a basis of the space of homogeneous polynomial functions of a given degree on the group of orthogonal similitudes. This will be applied in future work to the orthogonal Schur algebra, as defined by Doty [Dot].

In the case of the symplectic group, a basis involving bideterminants was given by Oehms [O] for the space of homogeneous polynomial functions of a given degree on the group of symplectic similitudes. Our methods are quite different from those of Oehms.

Filtrations for the coordinate ring of the symplectic group were also considered by de Concini $[\mathrm{dC}]$. Filtrations of this sort have been studied by Donkin and by Kopppinen $[\mathrm{K}]$ for connected reductive groups $G$. The orthogonal group is not connected, and our emphasis is on explicit filtrations. For connected, simply connected semi-simple groups $G$, the coordinate ring $\mathbb{C}[G]$ has a canonical basis, due to Lusztig, [L], Chapter 29. This basis is not explicitly given.

The author would like to thank V. Chernousov, A. Pianzola, and the referee, for comments especially concerning Section 7.

\section{PRELiminaries}

Let $n \geq 3$ be a positive integer and let $m$ be the greatest integer $\leq n / 2$. Define the ordered set $\mathcal{I}=\{\overline{1}<1<\overline{2}<2<\cdots<\bar{m}<m\}$ if $n$ is even, and $\mathcal{I}=\{\overline{1}<1<\overline{2}<2<\cdots<\bar{m}<$ $m<0\}$ if $n$ is odd. Define

$$
\overline{\bar{\imath}}=i, \quad 1 \leq i \leq m, \quad \overline{0}=0 .
$$

Let $\left\{v_{i}: i \in \mathcal{I}\right\}$ be the standard basis of $V=K^{n}$, and define the symmetric bilinear form on $V$

$$
\left\langle\sum_{i \in \mathcal{I}} x_{i} v_{i}, \sum_{i \in \mathcal{I}} y_{i} v_{i}\right\rangle=\sum_{i \in \mathcal{I}} x_{i} y_{\bar{\imath}}=\sum_{i=1}^{m}\left(x_{i} y_{\bar{\imath}}+x_{\bar{\imath}} y_{i}\right)+x_{0} y_{0}
$$

where $x_{0}=0$ if $n$ is even. The orthogonal group $O(n, K)$, which we will also denote by $O(n)$, is defined as the subgroup of $G L(n, K)$ which preserves the form $\langle$,$\rangle given in (2.1).$

As usual, a partition $\lambda$ of a positive integer $r$ into $k$ parts is given by writing $r$ as a sum $r=\lambda_{1}+\lambda_{2}+\cdots+\lambda_{k}$ of positive integers where $\lambda_{1} \geq \lambda_{2} \geq \cdots \geq \lambda_{k}$. We let $|\lambda|$ denote $r$, and we call $|\lambda|$ the size of $\lambda$. A Young tableau of shape $\lambda$ is a left justified array, having $k$ rows; the $i$-th row has $\lambda_{i}$ entries from the set $\mathcal{I}$. We will denote the $i$-th column of $T$ by $T_{i}$.

The conjugate $\lambda^{\prime}$ of a partition $\lambda$ is the partition whose parts are the column lengths of a tableau of shape $\lambda$.

The dominance order on partitions of $r$ is defined by $\lambda \unlhd \mu$ if $\lambda_{1}+\cdots+\lambda_{i} \leq \mu_{1}+\cdots+\mu_{i}$ for all $i \in\{1, \ldots, k\}$, where $\lambda$ has $k$ parts. (For this to make sense we let $\mu_{j}=0$ if $j$ is greater than the number of parts of $\mu$.) For example, if $\lambda=(2,2,1)$ and $\mu=(4,1)$, then $\lambda \unlhd \mu$. We will use the following elementary result.

Remark 2.1. If $T$ is a tableau of shape $\lambda$ and the tablea $+u S$ is obtained from $T$ by moving an entry from the bottom of a column to the bottom of a column to the left, then the shape $\mu$ of $S$ satisfies $\mu \triangleleft \lambda$.

A Young tableau is called $G L(n)$-standard if it has at most $n$ rows, and if the entries are non-decreasing from left to right across each row and strictly increasing from top to bottom 
down each column. We will give the definition, due to $[\mathrm{KW}]$, of what is called an $O(n)$ standard Young tableau $T$. For $i=1,2, \ldots, m$ let $\alpha_{i}$ and $\beta_{i}$ be the number of entries less than or equal to $i$ in the first and second columns, respectively, of $T$. Let $T(i, j)$ denote the entry in row $i$ and column $j$ of $T$. We shall call $T O(n)$-standard if it is $G L(n)$-standard, if $\lambda_{1}^{\prime}+\lambda_{2}^{\prime} \leq n$, and if in addition, for each $i=1,2, \ldots, m$,

(OS 1) $\alpha_{i}+\beta_{i} \leq 2 i$

$(\mathrm{OS} 2)$ if $\alpha_{i}+\beta_{i}=2 i$ with $\alpha_{i}>\beta_{i}$ and $T\left(\alpha_{i}, 1\right)=i$ and $T\left(\beta_{i}, 2\right)=\bar{\imath}$ then $T\left(\alpha_{i}-1,1\right)=\bar{\imath}$;

(OS 3) if $\alpha_{i}+\beta_{i}=2 i$ with $\alpha_{i}=\beta_{i}(=i)$ and if $\bar{\imath}, i$ occur in the $i$-th row of $T$, with $\bar{\imath}$ in $T_{1}$ and $i$ in $T_{b}$ for some $b \geq 2$, then above the $i$ in $T_{b}$ there is an $\bar{l}$.

In cases (2) and (3), the entry $i$ is said to be protected by the existence of $\bar{\imath}$ above it.

For each pair $(i, j)$ where $i$ and $j$ are in $\mathcal{I}$, let $X(i, j)$ be an indeterminate, and let $X=(X(i, j))$ be the $n \times n$ matrix whose rows and columns are indexed by $\mathcal{I}$, and whose $(i, j)$-entry is $X(i, j)$. Let $K[X(i, j)]$ be the polynomial ring in the $n^{2}$ indeterminates $X(i, j)$.

The coordinate ring $K[O(n)]$ consists of the restrictions of the functions in $K[X(i, j)]$ to the orthogonal group $O(n)$. (Strictly speaking, a function in $K[O(n)]$ should be defined as a function on $O(n)$ given by a polynomial in $K[X(i, j)]$ divided by some power of the determinant. Since det $= \pm 1$ on $O(n)$, then $1 / \operatorname{det}=\operatorname{det}$ as functions on $O(n)$; hence all functions in $K[O(n)]$ are given by polynomials.)

Suppose that $S$ and $T$ are tableaux of the same shape $\lambda$. Define $\left[S_{i}: T_{i}\right]$ to be the determinant of the submatrix of $X$ whose rows are indexed by the entries of the column $S_{i}$ and whose columns are indexed by the entries of the column $T_{i}$. Define the bideterminant, denoted $[S: T]$, to be the product

$$
[S: T]=\prod_{i=1}^{\lambda_{1}}\left[S_{i}: T_{i}\right] .
$$

We will sometimes use the notation

$$
[S: T]_{O}
$$

to denote the bideterminant $[S: T]$ considered as a function on $O(n)$. We define the shape of $[S: T]$ or of $[S: T]_{O}$ to be $\lambda$, which is the common shape of $S$ and $T$. We call $[S: T]_{O}$ an $O(n)$-standard bideterminant if both $S$ and $T$ are $O(n)$-standard.

For an $n \times n$ matrix $A$, let $A^{t}$ denote the transpose of $A$.

Remark 2.2. The bideterminant $[S: T]$ evaluated at $X^{t}$ is equal to the bideterminant $[T: S]$.

For $1 \leq k \leq n$, let

$$
j_{1}, j_{2}, \ldots, j_{k}
$$

denote the first $k$ elements of the ordered set $\mathcal{I}$. For example, if $k=3$, then $j_{1}=\overline{1}, j_{2}=1$, $j_{3}=\overline{2}$. The basic $\lambda$-tableau, denoted $T^{\lambda}$, is the tableau having each entry in row $k$ equal to $j_{k}$.

We define the following partial order on the set of tableaux of shape $\lambda$. If $T \neq T^{\prime}$, suppose that in the right-most column in which there is a differing entry, the top-most entry $i$ of $T$ which differs from an entry $i^{\prime}$ of $T^{\prime}$ in the same position satisfies $i<i^{\prime}$; then we say that $T \prec T^{\prime}$.

By a signed sum of some quantities $x_{1}, x_{2}, \ldots, x_{k}$ we mean a linear combination $\sum_{i=1}^{k} \epsilon_{i} x_{i}$ where each $\epsilon_{i} \in\{-1,1\}$. 
We say that $i, \bar{\imath}$ occur in a 2-column tableau $T$ if $i$ is an entry in the first column of $T$ and $\bar{\imath}$ is an entry in the second. To delete the pair $i, \bar{\imath}$, remove $i$ from the first column and $\bar{\imath}$ from the second, and move up the other entries to form a tableau.

We will let $\# A$ denote the size of a finite set $A$.

\section{3. $G L(n)$-Straightening}

Writing a bideterminant as a linear combination of $G L(n)$-standard ones is often called straightening. We shall describe the straightening method used by Mead [M].

Suppose that $S$ and $T$ are tableaux of the same shape $\lambda$ having two columns, of lengths $k$ and $\ell$ respectively. Suppose that $S$ is column-increasing but not $G L(n)$-standard. Using Mead's notation for the entries of the tableaux (although Mead does not use tableaux or the term bideterminant) suppose that

$$
[S: T]=\left[\begin{array}{ccccc}
i_{1} & i_{1}^{\prime} & & a_{1} & b_{1} \\
\vdots & \vdots & & \vdots & \vdots \\
i_{\ell} & i_{\ell}^{\prime} & \vdots & a_{\ell} & b_{\ell} \\
\vdots & & \vdots & \\
i_{k} & & & a_{k} &
\end{array}\right]
$$

Suppose that $i_{j} \leq i_{j}^{\prime}$ for $j<t$ but $i_{t}>i_{t}^{\prime}$.

Let $H$ be the $(k+\ell) \times(k+\ell)$ matrix given by

$$
H=\left(\begin{array}{cc}
B_{1} & C \\
D & B_{2}
\end{array}\right)
$$

where $B_{1}$ is the $k \times k$ submatrix of $X=(X(i, j))$ whose rows and columns are indexed by $S_{1}$ and $T_{1}$ respectively, $B_{2}$ is the $\ell \times \ell$ submatrix of $X$ whose rows and columns are indexed by $S_{2}$ and $T_{2}$, respectively, $C$ is the $k \times \ell$ matrix defined by

$$
c_{p, q}=0 \text { if } p<t, \quad c_{p, q}=X\left(i_{p}, b_{q}\right) \text { if } p \geq t
$$

and $D$ is the $\ell \times k$ matrix given by

$$
d_{p, q}=X\left(i_{p}^{\prime}, a_{q}\right) \text { if } p \leq t, \quad d_{p, q}=0 \text { if } p>t .
$$

Use Laplace expansion along multiple rows or columns (see, for example, [Pra, 2.4.1, p. 11]), in two ways, as follows: expand $\operatorname{det} H$ by minors of the first $k$ columns, and by minors of $\ell-1$ rows consisting of the first $t-1$ rows along with the last $\ell-t$ rows. (An example will be given below.)

The bideterminant $[S: T]$ occurs as one of the terms of the first expansion; solve for $[S: T]$ equal to the negative of the signed sum of the other bideterminants from the first expansion plus the signed sum of bideterminants from the second expansion.

All the terms in the row-expansion involve bideterminants where the tableaux have 2 columns, of length $k+1, \ell-1$, respectively, hence of shape $\triangleleft \lambda$ by Remark 2.1 .

In the column expansion, each term is a product of two minors, the second of which has rows indexed by $e_{1}, e_{2}, \ldots, e_{k}, i_{t+1}^{\prime}, \ldots, i_{\ell}^{\prime}$ where $\left\{e_{1}, e_{2}, \ldots, e_{k}\right\}$ vary over all possible subsets of $\left\{i_{t}, \ldots, i_{k}, i_{1}^{\prime}, \ldots, i_{t}^{\prime}\right\}$. The term $[U: T]$ with $U$ the lowest (in the order $\prec$ ) is the one where $\left\{e_{1}, e_{2}, \ldots, e_{t}\right\}=\left\{i_{1}^{\prime}, \ldots, i_{t}^{\prime}\right\}$; this gives the bideterminant $[S: T]$. All the other bideterminants are of the form $[U: T]$ where $U \succ S$.

So we have the following result. 
Lemma 3.1. Let $S$ and $T$ be two-column tableaux of the same shape $\lambda$, where $S$ is column increasing but not $G L(n)$-standard. Then (i)

$$
[S: T]=\sum_{U} a_{U}[U: T]+s
$$

where each $U$ occurring in the sum is a tableau of shape $\lambda$ where $U \succ S$, each $a_{U} \in\{1,-1\}$, and $s$ is signed sum of bideterminants of shapes $\mu \triangleleft \lambda$. (ii) The tableaux $U$ in the sum, and the signs $a_{U}$ depend only on $S$ and not on $T$. (iii) If $T=T^{\lambda}$, then $s=0$.

Proof. We need only prove (ii) and (iii). For independence of $T$, the row indices in the column expansion of det $H$ depend only on $S$. For (iii), if the rows of $T$ all have equal entries, in the row expansion of det $H$, each bideterminant we get has the form $[V: W]$ where the first column of $V$ has repeated entries and so $[V: W]=0$.

Example 3.1. Consider the bideterminant

$$
\begin{aligned}
& {[S: T]=\left[\begin{array}{lllll}
1 & 1 & & \overline{1} & 1 \\
2 & \overline{2} & : & \overline{2} & 2 \\
3 & & & 3 &
\end{array}\right] .} \\
& \text { Then } H=\left(\begin{array}{ccccc}
X(1, \overline{1}) & X(1, \overline{2}) & X(1,3) & 0 & 0 \\
X(2, \overline{1}) & X(2, \overline{2}) & X(2,3) & X(2,1) & X(2,2) \\
X(3, \overline{1}) & X(3, \overline{2}) & X(3,3) & X(3,1) & X(3,2) \\
X(1, \overline{1}) & X(1, \overline{2}) & X(1,3) & X(1,1) & X(1,2) \\
X(\overline{2}, \overline{1}) & X(\overline{2}, \overline{2}) & X(\overline{2}, 3) & X(\overline{2}, 1) & X(\overline{2}, 2)
\end{array}\right) \text {. }
\end{aligned}
$$

For the expansion of $\operatorname{det} H$ along the first three columns, we get terms which are a product of two minors: in the first minor, we use the first row, we omit the fourth row, since its first three entries are the same as those of the first row, and we have a choice of two of rows 2,3 , and 5. The second minor has the complementary rows. This gives

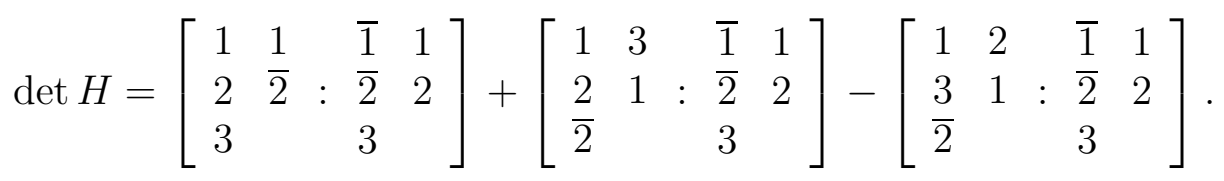

The row expansion is along the first row, giving three terms:

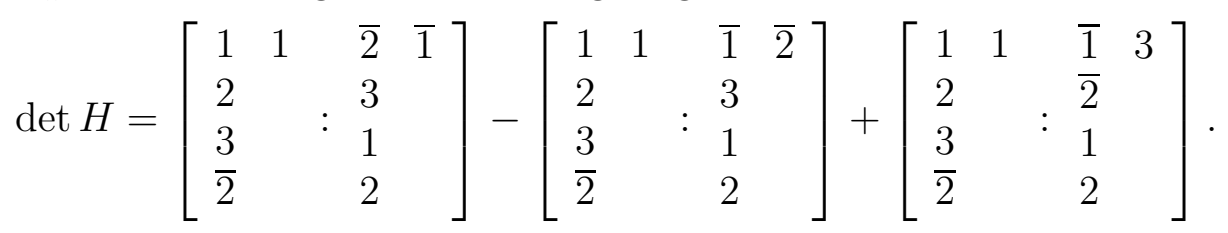

We switch rows to give standard tableaux. Then

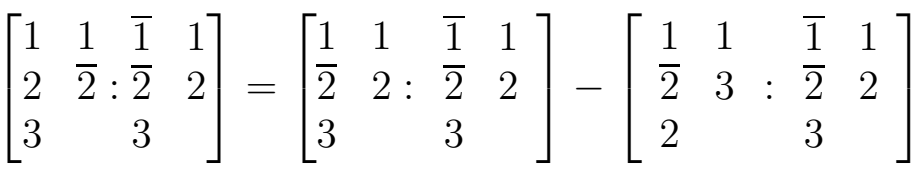

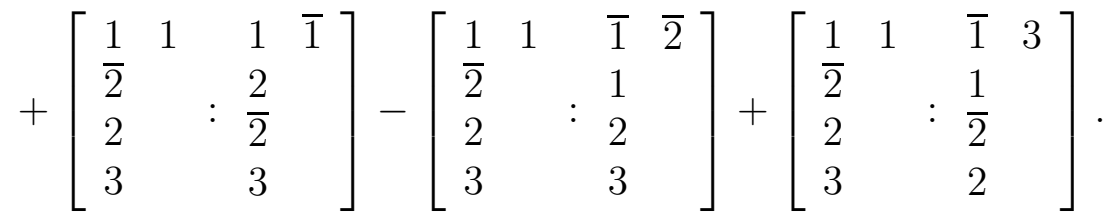

We have the following result on $G L(n)$-straightening. 
Theorem 3.1. Suppose that $S$ and $T$ are tableaux of the same shape $\lambda$. Then

$$
[S: T]=\sum_{U} b_{U}[U: T]+s
$$

where the tableaux $U$ in the sum are $G L(n)$-standard, each $b_{U} \in K$ and is independent of $T$, and $s$ is a signed sum of bideterminants of shapes $\mu \triangleleft \lambda$.

Proof. This is proved, using Lemma 3.1, by induction on the order $\triangleleft$ and downward induction on $\prec$.

Using Remark 2.2 there is an analogous version where the roles of $S$ and $T$ are interchanged, writing $[T: S]$ as a linear combination of $[T: U]$ plus bideterminants of shape smaller than $\lambda$ in the $\triangleleft$ order. This gives the spanning part of the following well-known result. For linear independence, which we do not need, see [M], [DRS] or [DEP].

Theorem 3.2. The bideterminants $[S: T]$ where both $S$ and $T$ are $G L(n)$-standard are a basis of the ring $K[X(i, j)], 1 \leq i, j \leq n$.

In Sections 7 and 8 we will replace $K$ with the $\operatorname{ring} \mathbb{Z}$ or $\mathbb{Z}[1 / 2]$. In the statement of Theorem 3.1, the coefficients $b_{U}$ come from the coefficients $a_{U}$ of Lemma 3.1, and these are \pm 1 . So we have the following.

Theorem 3.3. The bideterminants $[S: T]$ where both $S$ and $T$ are $G L(n)$-standard are a basis of the ring $R[X(i, j)], 1 \leq i, j \leq n$, where $R$ is any commutative ring.

As with Theorem 3.2 we will not need to use linear independence. We will also use the following.

Lemma 3.2. Suppose that $S$ and $T$ are tableaux of the same shape $\lambda$ having two columns, where $S$ is $G L(n)$-standard. Suppose that $\bar{\imath}, i$ occur in $S$, both in the $t$-th row, and let $S^{*}$ be the result of replacing this pair $\bar{\imath}, i$ with $i, \bar{\imath}$. Then

$$
\left[S^{*}: T\right]=[S: T]+\sum_{U} a_{U}[U: T]+s
$$

where each $U$ is a $\lambda$-tabeau such that $S^{*} \prec U$, each $a_{U}$ is \pm 1 , and $s$ is signed sum of bideterminants of shape $\mu$ where $\mu \triangleleft \lambda$. The $U$ and $a_{U}$ occurring in the equation are independent of $T$.

Proof. In Mead's method, in the column expansion, the lowest $U$ is $S$, and the second lowest is $S^{*}$.

This is illustrated in Example 3.1 above, where $[S: T]$ is written as a signed sum of standard bideterminants, the first of which is $\left[S^{*}: T\right]$ where $S^{*}$ is obtained from $S$ by replacing $2, \overline{2}$ in the second row by $\overline{2}, 2$, and and the second is $[U: T]$ where $U \succ S^{*}$.

\section{Main Technical Result}

As functions on $O(n)$, we have

$$
\sum_{i \in \mathcal{I}} X(i, j) X(\bar{\imath}, k)=\delta_{j \bar{k}}, \quad j, k \in \mathcal{I} .
$$

In bideterminant notation,

$$
X(i, j) X(\bar{\imath}, k)=[i \bar{\imath}: j k]
$$


So we have, on $O(n)$,

$$
\sum_{i \in \mathcal{I}}[i \bar{\imath}: j k]=\delta_{j \bar{k}}
$$

We will need more general versions of this. For example, suppose that $S$ is a tableau having 2 columns, of lengths $e$ and $f$, and that $T$ is a tableau of 2 columns, of lengths $e+1$ and $f+1$. It will follow from our results below that if $i, \bar{\imath}$ do not occur in $T$ for any $i \in \mathcal{I}$, then

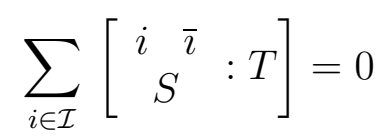

on $O(n)$. More generally, if $i, \bar{\imath}$ do occur in $T$ for some $i \in \mathcal{I}$, then

$$
\sum_{i \in \mathcal{I}}\left[\begin{array}{cc}
i{ }^{\bar{\imath}} & : T \\
S^{2} &
\end{array}\right]=\sum_{T^{\prime}} \pm\left[S: T^{\prime}\right]
$$

on $O(n)$, where the sum is over all tableaux $T^{\prime}$ obtained by deleting a pair $i, \bar{\imath}$ from $T$ if $i, \bar{\imath}$ occur in $T$.

For a more general version of this, we will have not just one variable $i$ in the summation, but $a$ variables $i_{1}, i_{2}, \ldots, i_{a}$, each of which will vary over $\mathcal{I}$, except that each $i_{j}$ will not be allowed to assume one of $c$ fixed values, where $0 \leq c<a$.

Suppose that $S$ and $T$ are tableaux of the same shape, having two columns. Suppose that $a$ is a positive integer, less than or equal to the length of the second column of $S$ (and of $T$ ). Suppose that $C$ is a (possibly empty) subset of $\mathcal{I}$ having $c$ elements, where $c<a$. Let $S_{0}$ be the tableau obtained from $S$ by deleting its first $a$ rows. We want to calculate the sum

$$
L=\sum_{i_{1}, \ldots, i_{a} \in \mathcal{I}-C}\left[\begin{array}{ccc}
i_{1} & \bar{\imath}_{1} & \\
i_{2} & \bar{\imath}_{2} & \\
\vdots & \vdots & : T \\
i_{a} & \bar{\imath}_{a} & \\
S_{0} &
\end{array}\right]
$$

We allow repeated indices in the sum, although a bideterminant having repeated indices $i_{s}$, is 0 .

Suppose that $d$ is an integer, $1 \leq d \leq a$, and suppose that $E=\left\{i_{1}, \ldots, i_{d}\right\}$ is a subset of $\mathcal{I}$ such that $i, \bar{\imath}$ occur in $T$ for all $i \in E$. Let

$$
(T, E)
$$

be the tableau obtained by deleting the pairs $i, \bar{\imath}$ from $T$ for each $i \in E$. Let $\mathcal{E}_{d}$ denote the set of all subsets $E$ of $\mathcal{I}$ of size $d$ such that $\bar{\imath}, i$ occur in $T$ for all $i \in E$.

We next define $\mathcal{S}_{d}$ by the equation

$$
\mathcal{S}_{d}=\sum_{E \in \mathcal{E}_{d}} \sum_{\substack{i_{1}<\cdots<i_{a-d} \\
i_{j} \in C}}(-1)^{a-d}\left[\begin{array}{ccc}
i_{1} & \bar{\imath}_{1} & \\
i_{2} & \bar{\imath}_{2} & \\
\vdots & \vdots & :(T, E) \\
i_{a-d} & \bar{l}_{a-d} & \\
\multicolumn{2}{c}{S_{0}} &
\end{array}\right]
$$

If for a given $d, C$ has fewer than $a-d$ elements, then the second sum is the empty sum, and $\mathcal{S}_{d}=0$. In particular, if $C$ is empty, then each $\mathcal{S}_{d}=0$ except for $d=a$. 
Lemma 4.1. With the above notation, the sum $L$ in [4.1), as function on $O(n)$, is given by

$$
L=a ! \sum_{d=1}^{a} \mathcal{S}_{d} .
$$

Before giving the proof, we have an example. Consider

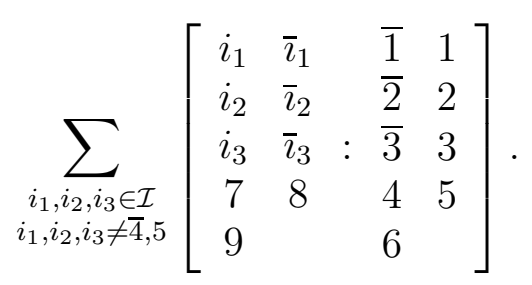

Then $a=3, C=\{\overline{4}, 5\}, c=\# C=2$. First suppose that $d=1$. Since $a-d=2$, there is a unique subset of $C$ of size 2 , so we replace $\left(i_{1}, \bar{\imath}_{1}\right),\left(i_{2}, \bar{\imath}_{2}\right),\left(i_{3}, \bar{\imath}_{3}\right)$ with the two pairs $(\overline{4}, 4),(5, \overline{5})$. There are three subsets $E_{1}$.

$$
\mathcal{S}_{1}=\left[\begin{array}{llll}
\overline{4} & 4 & \overline{2} & 2 \\
5 & \overline{5} & \overline{3} & 3 \\
7 & 8 & 4 & 5 \\
9 & & 6 &
\end{array}\right]+\left[\begin{array}{llll}
\overline{4} & 4 & \overline{1} & 1 \\
5 & \overline{5} & \overline{3} & 3 \\
7 & 8 & 4 & 5 \\
9 & & 6 &
\end{array}\right]+\left[\begin{array}{llll}
\overline{4} & 4 & \overline{1} & 1 \\
5 & \overline{5} & \overline{2} & 2 \\
7 & 8 & 4 & 5 \\
9 & & 6 &
\end{array}\right]
$$

When $d=2$, we replace the pairs $\left(i_{1}, \bar{\imath}_{1}\right),\left(i_{2}, \bar{\imath}_{2}\right),\left(i_{3}, \bar{\imath}_{3}\right)$ from $S$ with $a-d=1$ pair $i, \bar{\imath}$ where $i \in C$. There are two ways to do this, since $C$ has two elements. There are three subsets $E_{2}$. So

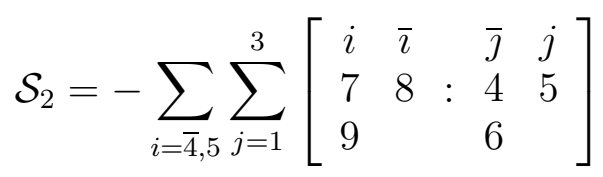

When $d=3$, there is only one $E_{3}$, namely $\{1,2,3\}$. Since $a-d=0$,

$$
\mathcal{S}_{3}=\left[\begin{array}{lllll}
7 & 8 & & 4 & 5 \\
9 & & & 6 &
\end{array}\right]
$$

Proof. Suppose that the entries of the two columns of $T$ are

$$
g_{1}, g_{2}, \ldots, g_{e}, \quad h_{1}, h_{2}, \ldots, h_{f}, \text { respectively. }
$$

Suppose further that the entries of the two columns of $S_{0}$ are

$$
j_{a+1}, \ldots, j_{e}, \quad k_{a+1}, \ldots, k_{f} \text { respectively. }
$$

Let $P$ be the permutations of the symbols $g_{1}, g_{2}, \ldots, g_{e}$, and $Q$ the permutations of the symbols $h_{1}, \ldots, h_{f}$.

Then the sum $L$ in (4.1) is given by

$$
\begin{aligned}
L=\sum_{i_{1}, \ldots, i_{a} \in \mathcal{I}-C} \sum_{\sigma \in P} \operatorname{sgn}(\sigma) \prod_{s=1}^{a} X\left(i_{s}, \sigma\left(g_{s}\right)\right) & \prod_{s=a+1}^{e} X\left(j_{s}, \sigma\left(g_{s}\right)\right) \\
& \times \sum_{\tau \in Q} \operatorname{sgn}(\tau) \prod_{t=1}^{a} X\left(\bar{\imath}_{t}, \tau\left(h_{t}\right)\right) \prod_{t=a+1}^{f} X\left(k_{t}, \tau\left(h_{t}\right)\right) .
\end{aligned}
$$


A BASIS OF BIDETERMINANTS FOR THE COORDINATE RING OF THE ORTHOGONAL GROUP 9

Define

Then we have

$$
Y_{\sigma, \tau}=\prod_{s=a+1}^{e} X\left(j_{s}, \sigma\left(g_{s}\right)\right) \prod_{t=a+1}^{f} X\left(k_{t}, \tau\left(h_{t}\right)\right)
$$

$$
L=\sum_{\sigma \in P} \sum_{\tau \in Q} \operatorname{sgn}(\sigma) \operatorname{sgn}(\tau) \prod_{s=1}^{a}\left(\sum_{i_{s} \in \mathcal{I}-C} X\left(i_{s}, \sigma\left(g_{s}\right)\right) X\left(\bar{l}_{s}, \tau\left(h_{s}\right)\right)\right) Y_{\sigma, \tau} .
$$

Since $\sum_{i \in \mathcal{I}} X(i, j) X(\bar{\imath}, k)=\delta_{j, \bar{k}}$ on $O(n)$, then as functions on $O(n)$, for each $s$ in $\{1, \ldots, a\}$,

$$
\sum_{i_{s} \in \mathcal{I}-C} X\left(i_{s}, \sigma\left(g_{s}\right)\right) X\left(\bar{\imath}_{s}, \tau\left(h_{s}\right)\right)=\delta_{\sigma\left(g_{s}\right), \overline{\tau\left(h_{s}\right)}}-\sum_{i_{s} \in C} X\left(i_{s}, \sigma\left(g_{s}\right)\right) X\left(\bar{\imath}_{s}, \tau\left(h_{s}\right)\right) .
$$

Hence as a function on $O(n), L$ is equal to

$$
L^{\prime}=\sum_{\sigma \in P} \sum_{\tau \in Q} \operatorname{sgn}(\sigma) \operatorname{sgn}(\tau) \prod_{s=1}^{a}\left(\delta_{\sigma\left(g_{s}\right), \overline{\tau\left(h_{s}\right)}}-\sum_{i_{s} \in C} X\left(i_{s}, \sigma\left(g_{s}\right)\right) X\left(\bar{\imath}_{s}, \tau\left(h_{s}\right)\right)\right) Y_{\sigma, \tau} .
$$

To simplify the notation somewhat, fix $(\sigma, \tau) \in P \times Q$, and let

$$
\delta(s)=\delta_{\sigma\left(g_{s}\right), \overline{\tau\left(h_{s}\right)}}, \quad Z\left(s, i_{s}\right)=X\left(i_{s}, \sigma\left(g_{s}\right)\right) X\left(\bar{\imath}_{s}, \tau\left(h_{s}\right)\right) .
$$

With this notation we have, for fixed $\sigma, \tau$,

$$
\prod_{s=1}^{a}\left(\delta_{\sigma\left(g_{s}\right), \overline{\tau\left(h_{s}\right)}}-\sum_{i_{s} \in C} X\left(i_{s}, \sigma\left(g_{s}\right)\right) X\left(\bar{\imath}_{s}, \tau\left(h_{s}\right)\right)\right)=\prod_{s=1}^{a}\left(\delta(s)-\sum_{i_{s} \in C} Z\left(s, i_{s}\right)\right) .
$$

Expand the product

$$
\prod_{s=1}^{a}\left(\delta(s)-\sum_{i_{s} \in C} Z\left(s, i_{s}\right)\right) .
$$

To do this, for each $s$ select a term from the factor $\left(\delta(s)-\sum_{i_{s} \in C} Z\left(s, i_{s}\right)\right)$, namely $\delta(s)$ or $-Z\left(s, i_{s}\right)$ for some $i_{s} \in C$; multiply all the chosen terms, and sum over all possible choices of terms. For a given choice, let $D$ be the set of all $s$ for which we pick $\delta(s) \neq 0$. If $s \in D$, since

then

$$
0 \neq \delta(s)=\delta_{\sigma\left(g_{s}\right), \overline{\tau\left(h_{s}\right)}}
$$

$$
\sigma\left(g_{s}\right)=\overline{\tau\left(h_{s}\right)}
$$

Let $\mathcal{D}$ be the set of all subsets $D$, including the empty subset, of $\{1, \ldots, a\}$ such that $\delta(s) \neq 0$ for all $s \in D$. For a given $D \in \mathcal{D}$, let

$$
\ell=\ell(D)=a-\# D
$$

and let $D^{\prime}$ be the complement of $D$ in $\{1,2, \ldots, a\}$. Then

$$
\prod_{s=1}^{a}\left(\delta(s)-\sum_{i_{s} \in C} Z\left(s, i_{s}\right)\right)=\sum_{D \in \mathcal{D}}(-1)^{\ell(D)} \sum_{\left(i_{1}, \ldots, i_{\ell}\right) \in C^{\ell}} \prod_{s \in D^{\prime}} Z\left(s, i_{s}\right) .
$$

Write $\mathcal{D}=\mathcal{D}(\sigma, \tau)$. Then as functions on $O(n), L$ is equal to

$$
L^{\prime \prime}=\sum_{\substack{\sigma \in P \\ \tau \in Q}} \operatorname{sgn}(\sigma) \operatorname{sgn}(\tau) \sum_{D \in \mathcal{D}(\sigma, \tau)}(-1)^{\ell(D)} \sum_{\left(i_{1}, \ldots, i_{\ell}\right) \in C^{\ell}} \prod_{s \in D^{\prime}} X\left(i_{s}, \sigma\left(g_{s}\right)\right) X\left(\bar{\imath}_{s}, \tau\left(h_{s}\right)\right) Y_{\sigma, \tau} .
$$


We want to interchange the order of the first two summations. Let $\mathcal{P}$ denote the set of all subsets of $\{1, \ldots, a\}$. For a given subset $D$ of $\mathcal{P}$, define

$$
G(D)=\left\{(\sigma, \tau) \in P \times Q: \sigma\left(g_{s}\right)=\overline{\tau\left(h_{s}\right)} \text { for all } s \in D\right\} .
$$

If $D$ is empty, then $G(D)=P \times Q$. Then

$$
L^{\prime \prime}=\sum_{D \in \mathcal{P}}(-1)^{\ell(D)} \sum_{(\sigma, \tau) \in G(D)} \operatorname{sgn}(\sigma) \operatorname{sgn}(\tau) \sum_{\left(i_{1}, \ldots, i_{\ell}\right) \in C^{\ell}} \prod_{s \in D^{\prime}} X\left(i_{s}, \sigma\left(g_{s}\right)\right) X\left(\bar{\imath}_{s}, \tau\left(h_{s}\right)\right) Y_{\sigma, \tau} .
$$

For a given subset $D$ of $\mathcal{P}$, let

$$
L^{\prime \prime}(D)=\sum_{(\sigma, \tau) \in G(D)} \operatorname{sgn}(\sigma) \operatorname{sgn}(\tau) \sum_{\left(i_{1}, \ldots, i_{\ell}\right) \in C^{\ell}} \prod_{s \in D^{\prime}} X\left(i_{s}, \sigma\left(g_{s}\right)\right) X\left(\bar{l}_{s}, \tau\left(h_{s}\right)\right) Y_{\sigma, \tau} .
$$

Hence

$$
L^{\prime \prime}=\sum_{D \in \mathcal{P}}(-1)^{\ell(D)} L^{\prime \prime}(D) .
$$

Let $\mathcal{P}_{d}$ be the set of subsets of $\{1, \ldots, a\}$ of size $d$, and let

$$
L_{d}^{\prime \prime}=\sum_{D \in \mathcal{P}_{d}}(-1)^{a-d} L^{\prime \prime}(D) ; \quad \text { then } \quad L^{\prime \prime}=\sum_{d=0}^{a} L_{d}^{\prime \prime} .
$$

For a fixed $D \in \mathcal{P}_{d}$, let $\mathbf{e}$ be a $d$-tuple $\left\{e_{1}, e_{2}, \ldots, e_{d}\right\}$ of distinct elements of $\mathcal{I}$. Let

$$
P(D, \mathbf{e})=\left\{\sigma \in P: \sigma\left(g_{s}\right)=e_{s}, s \in D\right\}, Q(D, \mathbf{e})=\left\{\tau \in Q: \tau\left(h_{s}\right)=\bar{e}_{s}, s \in D\right\} .
$$

If $D$ is empty, then $\mathbf{e}$ is a 0 -tuple, and then $P(D, \mathbf{e})=P$, and $Q(D, \mathbf{e})=Q$. Let

$$
L^{\prime \prime}(D, \mathbf{e})=\sum_{\substack{\sigma \in P(D, \mathbf{e}) \\ \tau \in Q(D, \mathbf{e})}} \operatorname{sgn}(\sigma) \operatorname{sgn}(\tau) \sum_{\left(i_{1}, \ldots, i_{\ell}\right) \in C^{\ell}} \prod_{s \in D^{\prime}} X\left(i_{s}, \sigma\left(g_{s}\right)\right) X\left(\bar{\imath}_{s}, \tau\left(h_{s}\right)\right) Y_{\sigma, \tau} .
$$

Let $\mathcal{F}_{d}$ denote the set of all $d$-tuples of distinct elements of $\mathcal{I}$; then

$$
L^{\prime \prime}(D)=\sum_{\mathbf{e} \in \mathcal{F}_{d}} L^{\prime \prime}(D, \mathbf{e}) .
$$

For a given $D$ and $\mathbf{e}$, consider a fixed $\ell$-tuple $(\ell=\ell(D))$

$$
\mathbf{i}=\left(i_{1}, \ldots, i_{\ell}\right) \in C^{\ell} \text {. }
$$

For this i let

so

$$
L^{\prime \prime}(D, \mathbf{e}, \mathbf{i})=\sum_{(\sigma, \tau) \in P(D, \mathbf{e}) \times Q(D, \mathbf{e})} \operatorname{sgn}(\sigma) \operatorname{sgn}(\tau) \prod_{s \in D^{\prime}} X\left(i_{s}, \sigma\left(g_{s}\right)\right) X\left(\bar{\imath}_{s}, \tau\left(h_{s}\right)\right) Y_{\sigma, \tau},
$$

$$
L^{\prime \prime}(D, \mathbf{e})=\sum_{\mathbf{i} \in C^{\ell}} L^{\prime \prime}(D, \mathbf{e}, \mathbf{i}) .
$$

Since

$$
Y_{\sigma, \tau}=\prod_{s=a+1}^{e} X\left(j_{s}, \sigma\left(g_{s}\right)\right) \prod_{t=a+1}^{f} X\left(k_{t}, \tau\left(h_{t}\right)\right)
$$

then $L^{\prime \prime}(D, \mathbf{e}, \mathbf{i})$ can be factored as the product $M(D, \mathbf{e}, \mathbf{i}) N(D, \mathbf{e}, \mathbf{i})$ where

$$
M(D, \mathbf{e}, \mathbf{i})=\sum_{\sigma \in P(D, \mathbf{e})} \operatorname{sgn}(\sigma) \prod_{s \in D^{\prime}} X\left(i_{s}, \sigma\left(g_{s}\right)\right) \prod_{s=a+1}^{e} X\left(j_{s}, \sigma\left(g_{s}\right)\right),
$$


A BASIS OF BIDETERMINANTS FOR THE COORDINATE RING OF THE ORTHOGONAL GROUP 11

$$
N(D, \mathbf{e}, \mathbf{i})=\sum_{\tau \in Q(D, \mathbf{e})} \operatorname{sgn}(\tau) \prod_{s \in D^{\prime}} X\left(\bar{\imath}_{s}, \tau\left(h_{s}\right)\right) \prod_{s=a+1}^{f} X\left(k_{s}, \tau\left(h_{s}\right)\right) .
$$

Up to a sign, $M(D, \mathbf{e}, \mathbf{i})$ is the determinant of the submatrix of $X$ whose rows are indexed by $i_{1}, \ldots, i_{\ell}$ (where $\mathbf{i}=\left(i_{1}, \ldots, i_{\ell}\right)$ ) and $j_{a+1}, \ldots, j_{e}$, and whose columns are indexed by the first column of $T$ with the set $E$ of entries of e deleted. Further, up to the same sign, $N(D, \mathbf{e}, \mathbf{i})$ is the determinant of the matrix whose rows are indexed by $\bar{\imath}_{1}, \ldots \bar{\imath}_{\ell}$ and $k_{a+1}, \ldots, k_{f}$, and whose columns are indexed by the second column of $T$, with $\bar{E}$ deleted. The product of the two signs is 1 , so

$$
L^{\prime \prime}(D, \mathbf{e}, \mathbf{i})=\left[\begin{array}{ccc}
i_{1} & \bar{\imath}_{1} & \\
i_{2} & \bar{\imath}_{2} & \\
\vdots & \vdots & :(T, E) \\
i_{\ell} & \bar{\imath}_{\ell} & \\
\multicolumn{2}{c}{S_{0}} &
\end{array}\right]
$$

This is one of the terms in equation (4.2). Note that the right side of (4.5) does not depend on $D$. Since there are $\left(\begin{array}{l}a \\ d\end{array}\right)$ subsets of $\{1, \ldots, a\}$ of size $d$, then for any $d$, pick some $D_{d} \in \mathcal{P}_{d}$, and then we have

$$
L_{d}^{\prime \prime}=\left(\begin{array}{l}
a \\
d
\end{array}\right) \sum_{\mathbf{e} \in \mathcal{F}_{d}}(-1)^{a-d} L^{\prime \prime}\left(D_{d}, \mathbf{e}\right) .
$$

Also, the right side of equation (4.5) does not depend on the ordering of the $d$-tuple e, just on the set $E$ of its entries. Write $L^{\prime \prime}(D, E)=L^{\prime \prime}(D, \mathbf{e})$. Each subset $E$ of $\mathcal{I}$ of size $d$ gives us $d$ ! distinct $d$-tuples in $\mathcal{F}_{d}$ by permuting the elements. Recall that $\mathcal{E}_{d}$ is the set of all subsets of $\mathcal{I}$ of size $d$. So we have

$$
L_{d}^{\prime \prime}=\left(\begin{array}{l}
a \\
d
\end{array}\right) d ! \sum_{E \in \mathcal{E}_{d}}(-1)^{a-d} L^{\prime \prime}\left(D_{d}, E\right)
$$

If $\mathbf{i}$ has two equal entries, then the right side of (4.5) is 0 , so $L^{\prime \prime}(D, E, \mathbf{i})=0$; hence we assume that all the entries of $\mathbf{i}$ are distinct. We did not exclude the possiblity that $D$ is the empty set. If it is, then $\ell(D)=a$, so $i_{1}, \ldots, i_{\ell}$ are $a$ elements of $C$, but we are assuming that $\# C<a$. So if $D$ is the empty set, then $\mathbf{i}$ has repeated entries, which we have excluded. Hence we may assume that $d \geq 1$, and it follows that

$$
L^{\prime \prime}=\sum_{d=1}^{a} L_{d}^{\prime \prime}
$$

If we permute the elements of $\mathbf{i}$, giving say $\mathbf{i}^{\prime}$, then $M\left(D, \mathbf{e}, \mathbf{i}^{\prime}\right)$ and $N\left(D, \mathbf{e}, \mathbf{i}^{\prime}\right)$ are, up to the same sign, equal to $M(D, \mathbf{e}, \mathbf{i})$ and $N(D, \mathbf{e}, \mathbf{i})$, respectively. So $L^{\prime \prime}\left(D, E, \mathbf{i}^{\prime}\right)=L^{\prime \prime}(D, E, \mathbf{i})$. Let

Then

$$
C(\ell)=\left\{\left(i_{1}, \ldots, i_{\ell}\right): i_{1}<\ldots<i_{\ell}, i_{j} \in C, j=1, \ldots, \ell\right\} .
$$

$$
L^{\prime \prime}(D, E)=\sum_{\mathbf{i} \in C^{\ell}} L^{\prime \prime}(D, E, \mathbf{i})=\ell ! \sum_{\mathbf{i} \in C(\ell)} L^{\prime \prime}(D, E, \mathbf{i}) .
$$

Since $\ell=a-d$ then

$$
L_{d}^{\prime \prime}=\left(\begin{array}{l}
a \\
d
\end{array}\right) d !(a-d) ! \sum_{E \in \mathcal{E}_{d}} \sum_{\mathbf{i} \in C(\ell)} L^{\prime \prime}(D, E, \mathbf{i})
$$


Since $\left(\begin{array}{l}a \\ d\end{array}\right) d !(a-d) !=a !$, then

$$
L_{d}^{\prime \prime}=\sum_{E \in \mathcal{D}_{d}} L(D, E)=a ! \sum_{d=1}^{a} \mathcal{S}_{d}
$$

Since $L=L^{\prime \prime}$ as functions on $O(n)$, the proof is complete.

In the definition of $L$ in (4.1) we allowed all possible $i_{1}, \ldots, i_{a} \in \mathcal{I}-C$. We define

$$
\widetilde{L}=\sum_{\substack{i_{1}, \ldots, i_{a} \in \mathcal{I}-C \\
i_{1}<\cdots<i_{a}}}\left[\begin{array}{ccc}
i_{1} & \bar{l}_{1} \\
i_{2} & \bar{t}_{2} \\
\vdots & \vdots & : T \\
i_{a} & \bar{\imath}_{a} \\
S_{0} &
\end{array}\right]
$$

Lemma 4.2. If $K$ has characteristic 0 , then as functions on $O(n), \widetilde{L}$ is equal to $\sum_{d=1}^{a} \mathcal{S}_{d}$.

Proof. As we have seen, repeated indices contribute nothing to the sum $L$. The result now follows from Lemma 4.1 by dividing by $a$ !.

It will follow from our results in Section 7 that this result also holds at positive characteristic. However, because of our characteristic zero assumption in this last lemma, we will need to assume that $K$ has charactersistic 0 for most of our results until the end of Section 6.

In Lemmas 4.1 and 4.2 , we sum over $E \in \mathcal{E}_{d}$; if for all $i \in \mathcal{I}, i, \bar{\imath}$ do not occur in $T$, then each $\mathcal{E}_{d}$ is empty, and $\mathcal{S}_{d}=0$. We then have the following.

Lemma 4.3. Suppose that for all $i \in \mathcal{I}, i, \bar{\imath}$ do not occur in $T$. Then as functions on $O(n)$,

$$
\sum_{\substack{i_{1} \ldots, i_{a} \in \mathcal{I}-C \\
i_{1}<\cdots<i_{a}}}\left[\begin{array}{ccc}
i_{1} & \bar{\imath}_{1} \\
i_{2} & \bar{\imath}_{2} \\
\vdots & \vdots & : T \\
i_{a} & \bar{\imath}_{a} \\
S_{0} &
\end{array}\right]=0 .
$$

A first application is that on $O(n)$, any bideterminant is, up to sign, equal to a bideterminant of shape $\lambda$, where $\lambda_{1}^{\prime}+\lambda_{2}^{\prime} \leq n$. We will prove this now in the two-column case.

Lemma 4.4. Supppose that $[S: T]$ is a bideterminant whose shape consists of a single column. Let $\bar{S}^{\prime}$ denote the column-increasing tableau whose entries are $\{\bar{\imath} \in \mathcal{I}: i \notin S\}$. Then as functions on $O(n)$,

$$
[S: T]= \pm \operatorname{det} \cdot\left[\bar{S}^{\prime}: \bar{T}^{\prime}\right]
$$

Proof. We assume that each of $S$ and $T$ has no repeated entries. Suppose that $S$ has $k$ entries, and let $a=n-k$. Let $V$ be the one-column tableau of length $n$ whose entries are all the elements of $\mathcal{I}$. Consider the sum

$$
s=\sum_{\substack{i_{1}, \ldots, i_{a} \in \mathcal{I} \\
i_{1}<\cdots<i_{a}}}\left[\begin{array}{ccc}
i_{1} & \bar{\imath}_{1} & \\
i_{2} & \bar{\imath}_{2} & \\
\vdots & \vdots & : V \bar{T}^{\prime} \\
i_{a} & \bar{\imath}_{a} & \\
S & &
\end{array}\right] .
$$


Apply Lemma 4.2. Since the set $C$ is empty, each $\mathcal{S}_{d}$ is empty except for $d=a$. We delete a pairs $i, \bar{\imath}$ from the tableau with columns $V, \bar{T}^{\prime}$; this means we delete all the entries of $\bar{T}^{\prime}$, and what remains of $V$ is $T$. So we get, as functions on $O(n)$,

$$
s= \pm[S: T] .
$$

On the other hand, unless $i_{1}, \ldots, i_{a}$ all do not occur in $S$,

$$
\left[\begin{array}{cc}
i_{1} & \\
i_{2} & \\
\vdots & : V \\
i_{a} & \\
S &
\end{array}\right]=0
$$

So all terms in the sum (4.8) are 0 except when $i_{1}, i_{2}, \ldots, i_{a}$ are the complementary entries to $S$, in which case

$$
\left[\begin{array}{cc}
i_{1} & \\
i_{2} & \\
\vdots & : V \\
i_{a} & \\
S &
\end{array}\right]= \pm \operatorname{det,}\left[\begin{array}{ccc}
i_{1} & \bar{\imath}_{1} & \\
i_{2} & \bar{\imath}_{2} & \\
\vdots & \vdots & : V \bar{T}^{\prime} \\
i_{a} & \bar{l}_{a} & \\
S & &
\end{array}\right]= \pm \operatorname{det} \cdot\left[\bar{S}^{\prime}: \bar{T}^{\prime}\right]
$$

So we get $[S: T]= \pm \operatorname{det} \cdot\left[\bar{S}^{\prime}: \bar{T}^{\prime}\right]$, on $O(n)$, as desired.

Lemma 4.5. Suppose that $S$ and $T$ each have shape $\lambda$, where each of $S$ and $T$ have two columns. Suppose that $\lambda_{1}^{\prime}+\lambda_{2}^{\prime}>n$. Then $[S: T]$ is equal, up to sign, to a bideterminant $[\widetilde{S}: \widetilde{T}]$ where $\widetilde{S}$ and $\widetilde{T}$ have shape $\widetilde{\lambda}$, where each of $\widetilde{S}, \widetilde{T}$ have two columns, and $\widetilde{\lambda}_{1}^{\prime}+\widetilde{\lambda}_{2}^{\prime}<n$.

Proof. Apply Lemma 4.4 to each of $\left[S_{1}: T_{1}\right]$ and $\left[S_{2}: T_{2}\right]$, giving, on $O(n)$

$$
[S: T]= \pm \operatorname{det}^{2} \cdot\left[\bar{S}_{1}^{\prime}: \bar{T}_{1}^{\prime}\right]\left[\bar{S}_{2}^{\prime}: \bar{T}_{2}^{\prime}\right] \text {. }
$$

If $g \in O(n)$, then $\operatorname{det}(g)= \pm 1$, so we may delete the factor $\operatorname{det}^{2}$. Let $[\widetilde{S}: \widetilde{T}]$ be the bideterminant where $\widetilde{S}_{1}=\bar{S}_{2}^{\prime}, \widetilde{S}_{2}=\bar{S}_{1}^{\prime}, \widetilde{T}_{1}=\bar{T}_{2}^{\prime}, \widetilde{T}_{2}=\bar{T}_{1}^{\prime}$. The shape of $[\widetilde{S}: \widetilde{T}]$ is $\widetilde{\lambda}$ where $\widetilde{\lambda}_{1}^{\prime}=n-\lambda_{2}^{\prime}, \widetilde{\lambda}_{2}^{\prime}=n-\lambda_{1}^{\prime}$. Then $[S: T]= \pm[\widetilde{S}: \widetilde{T}]$. Since $\lambda_{1}^{\prime}+\lambda_{2}^{\prime}>n$ then

$$
\widetilde{\lambda}_{1}^{\prime}+\widetilde{\lambda}_{2}^{\prime}=2 n-\lambda_{1}^{\prime}-\lambda_{2}^{\prime}<n
$$

This completes the proof.

\section{5. $O(n)$-STRAIGHTENING}

We assume that $K$ has characteristic 0 . The following three lemmas are replacements of Lemma $3.6,3.7$, and 3.8 of $[\mathrm{KW}]$. Our situation is more complicated than in $[\mathrm{KW}]$, as we need tableaux of different shapes and sizes.

We first deal with violations of condition (OS 1 ) in the definition of $O(n)$-standard in Section 2.

Lemma 5.1. Suppose that $S$ and $T$ are $\lambda$-tableaux having two columns, and that $S$ is not $O(n)$-standard, in that $\alpha_{j}+\beta_{j}>2 j$ for some $j$. Then

$$
[S: T]=\sum_{U \in \mathcal{S}, U \neq S}-[U: T]+s
$$


where $\mathcal{S}$ is a set of tableaux all of shape $\lambda$ such that $S \prec U$, and $s$ is a signed sum of bideterminants of shapes $\mu$ with $|\mu|<|\lambda|$. The set $\mathcal{S}$ does not depend on $T$. If $T=T^{\lambda}$, then $s=0$.

Proof. Let $\mathcal{J}=\{i \in \mathcal{I}: i \leq j\}$, and $\mathcal{K}=\{i \in \mathcal{I}: i>j\}$; then $\# \mathcal{J}=2 j$. For a column $S_{k}$ of $S$, write $i \in S_{k}$ if $i$ occurs as an entry in column $S_{k}$. Let

$$
\begin{aligned}
& A=\left\{i \in \mathcal{J}: i \in S_{1} \text { and } \bar{\imath} \in S_{2}\right\} \\
& B=\left\{i \in \mathcal{J}: i \in S_{1} \text { or } \bar{\imath} \in S_{2} \text { but not both }\right\} \\
& C=\left\{i \in \mathcal{J}: i \notin S_{1}, \bar{\imath} \notin S_{2}\right\} .
\end{aligned}
$$

Then $\mathcal{J}$ is the disjoint union of $A, B$, and $C$. Let $a=\# A, b=\# B, c=\# C$. Then $a+b+c=\# \mathcal{J}=2 j$, and $\alpha_{j}+\beta_{j}=2 a+b$. Since we are assuming that $\alpha_{j}+\beta_{j}>2 j$, then $2 a+b>2 j$, so $a>c$.

Let $\mathcal{S}$ be the set of all tableaux obtained from $S$ as follows. For each $i \in A$, replace the pair $i, \bar{\imath}$ in $S$ by a pair $i^{\prime}, \bar{\imath}^{\prime}$ where $i^{\prime} \in \mathcal{I}-C$; do this in all possible ways, where the replacements are strictly increasing down the first column of the tableau.

For each $U \in \mathcal{S}$, let $U^{\prime}$ be the tableau obtained from $U$ by rearranging the elements in each column so that the replacements $i, i^{\prime}$ occur in the first $a$ rows. Then

$$
\sum_{U \in \mathcal{S}}[U: T]= \pm \sum_{U \in \mathcal{S}}\left[U^{\prime}: T\right]
$$

and this last sum has the form (4.6). From Lemma 4.2, the sum $\sum_{U \in \mathcal{S}}[U: T]$ is equal to a signed sum $s$ of bideterminants having shapes $\mu$ such that $|\mu|<|\lambda|$, and from Lemma 4.3, $s=0$ if $T=T^{\lambda}$. If for some $U \in \mathcal{S}$ a replacement $i^{\prime}$ is in $B$, then either $i^{\prime}$ occurs twice in $U_{1}$ or $\bar{\imath}^{\prime}$ occurs twice in $U_{2}$, so the bideterminant $[U: T]=0$. So the only non-zero bideterminants in the sum $\sum_{U \in \mathcal{S}}[U: T]$ are those for which all the replacements come from $A \cup \mathcal{K}$, since the replacements cannot come from $C$. One of these is the original $[S: T]$, where each pair $i, \bar{\imath}$ is replaced by itself. Any other $U \in \mathcal{S}$ has at least one replacement $i^{\prime}$ coming from $\mathcal{K}$, so $i^{\prime}>j$ and therefore $S \prec U$. Then

$$
[S: T]=-\sum_{U \in \mathcal{S}, U \neq S}[U: T]+s .
$$

and for each $U$ where $[U: T]$ occurs in this sum, $S \prec U$. The tableaux $U$ in this sum depend only on $S$ and not on $T$.

Example. Consider the bideterminant as a function on $O(6)$

$$
[S: T]=\left[\begin{array}{lllll}
\overline{1} & \overline{2} & & 1 & 2 \\
\overline{2} & 2 & : & \overline{2} & 3 \\
2 & & & \overline{3} &
\end{array}\right] .
$$

For the tableau $S, \alpha_{2}=3, \beta_{2}=2, \alpha_{2}+\beta_{2}>4$. For $j=2, A=\{\overline{2}, 2\}, B=\{\overline{1}\}, C=\{1\}$. Then $a=2, c=1$. From Lemma 4.2, on $O(6)$,

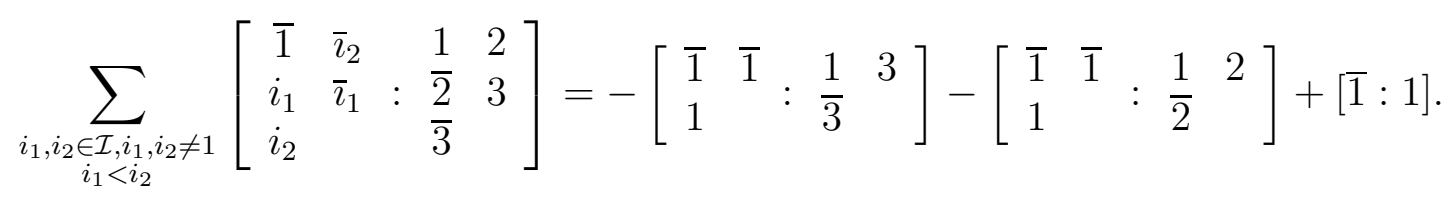


A BASIS OF BIDETERMINANTS FOR THE COORDINATE RING OF THE ORTHOGONAL GROUP 15

The sum on the left is equal to

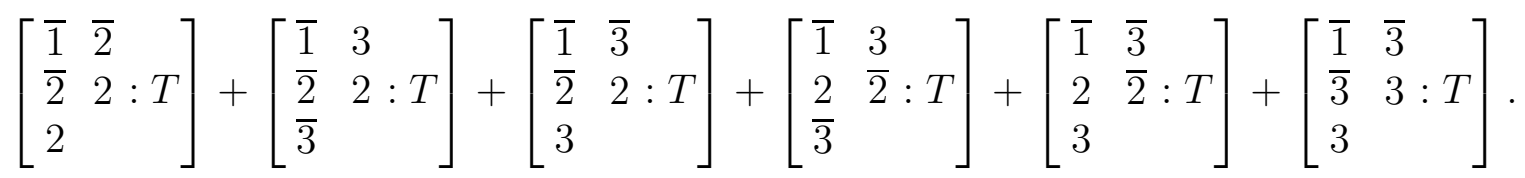

Then we get, on $O(6)$,

$$
\begin{aligned}
& {\left[\begin{array}{ll}
\overline{1} & \overline{2} \\
\overline{2} & 2
\end{array}: T\right]=-\left[\begin{array}{ll}
\overline{1} & 2 \\
\overline{2} & 3: T \\
\overline{3} &
\end{array}\right]-\left[\begin{array}{ll}
\overline{1} & 2 \\
\overline{2} & \overline{3}: T \\
3 &
\end{array}\right]-\left[\begin{array}{ll}
\overline{1} & \overline{2} \\
2 & 3: T \\
\overline{3} &
\end{array}\right]-\left[\begin{array}{ll}
\overline{1} & \overline{2} \\
2 & \overline{3}: T \\
3 &
\end{array}\right]} \\
& -\left[\begin{array}{lll}
\overline{1} & \overline{3} & \\
\overline{3} & 3 & : T \\
\overline{3} &
\end{array}\right]-\left[\begin{array}{lllll}
\overline{1} & \overline{1} & : & 1 & 3 \\
1 & & & \overline{3} &
\end{array}\right]-\left[\begin{array}{lllll}
\overline{1} & \overline{1} & : & 1 & 2 \\
1 & & & \overline{2} &
\end{array}\right]+[\overline{1}: 1] .
\end{aligned}
$$

Next we deal with violations of (OS 2)

Lemma 5.2. Suppose that $S$ and $T$ are column-increasing $\lambda$-tableaux with two columns, and that $S$ is not $O(n)$-standard, in that $\alpha_{j}+\beta_{j}=2 j$ for some $j$ with $\alpha_{j}>\beta_{j}$, and $S\left(\alpha_{j}, 1\right)=j, S\left(\beta_{j}, 2\right)=\bar{\jmath}, S\left(\alpha_{j}-1,1\right) \neq \bar{\jmath}$, Then $[S: T]$ may be expressed as a signed sum of bideterminants as in equation (5.1), Lemma 5.1.

Proof. Define $\mathcal{J}, \mathcal{K}, A, B, C, a, b, c$ as in the proof of Lemma 5.1. Since $j \in S_{1}$ and $\bar{\jmath} \in S_{2}$, then $j \in A$.

We claim that $\bar{\jmath} \in C$. If $\bar{\jmath}$ is in $S_{1}$, since $S_{\alpha_{j}, 1}=j$, we must have $\bar{\jmath}$ in position $\alpha_{j}-1$ of column 1, since the entries of the columns are strictly increasing. But we are assuming that $S\left(\alpha_{j}-1,1\right) \neq \bar{\jmath}$. So $\bar{\jmath}$ is not in column 1 of $S$. Since $S\left(\beta_{j}, 1\right)=\bar{\jmath}$, and $\bar{\jmath}<j$, then $j$ does not occur in $S_{2}$. So $\bar{\jmath} \in C$, as claimed.

Since $\alpha_{j}+\beta_{j}=2 j$, then $2 a+b=2 j$, and since $a+b+c=2 j$, then $a=c$. Let $A^{\prime}=A \cup\{\bar{\jmath}\}$, $C^{\prime}=C-\{\bar{\jmath}\}$, and let $a^{\prime}=\# A^{\prime}, c^{\prime}=\# C^{\prime}$, so $c^{\prime}<a$.

Now proceed with the argument of the proof of Lemma 5.1, but use $C^{\prime}$ instead of $C$ in the definition of $\mathcal{S}$. More precisely, let $\mathcal{S}^{\prime}$ be the set of all tableaux obtained from $S$ by replacing the pairs $i, \bar{\imath}$ in $S$ by $i^{\prime}, \bar{\imath}^{\prime}$ where $i^{\prime} \in \mathcal{I}-C^{\prime}$.

From Lemma 4.2, the sum $\sum_{U \in \mathcal{S}^{\prime}}[U: T]$ is equal to a signed sum of bideterminants having shapes $\mu$ with $|\mu|<|\lambda|$. One of the terms in the sum $\sum_{U \in \mathcal{S}^{\prime}}[U: T]$ is the original $[S: T]$. Of the others, all but one involve the replacement of at least one pair $i, \bar{\imath}$ with $i^{\prime}, \bar{\imath}^{\prime}$ where $i^{\prime} \in \mathcal{K}$, and so the resulting $U \succ S$. The one exception arises from the replacement of $j, \bar{\jmath}$ with $\bar{\jmath}, j$. The resulting $U \succ S$ since the second column of $U$ is identical to that of $S$ except that $\bar{\jmath}$ has been replaced by $j$, and $j>\bar{\jmath}$. This completes the proof.

Example. Consider the $O(7)$ bideterminant

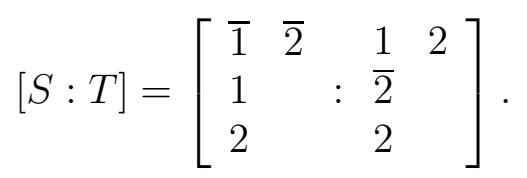

For $S$, with $j=2, \alpha_{2}+\beta_{2}=3+1=2 j$ and $\alpha_{2}>\beta_{2} ; S\left(\alpha_{2}, 1\right)=2, S\left(\beta_{2}, 2\right)=\overline{2}$, but the 2 in the first column of $S$ is not protected by a $\overline{2}$ above it. (Note that for $T$, the 2 in the first column is protected, and $T$ is $O(7)$-standard.) 
Using the notation of the lemma, applied to $S$, with $j=2$, we have $A=\{2\}, C=\{\overline{2}\}$, $C^{\prime}$ is empty. Let

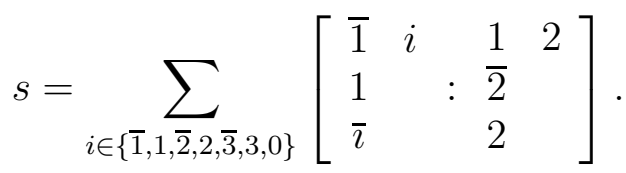

From Lemma 4.2, as functions on $O(7)$,

$$
s=\left[\begin{array}{lll}
\overline{1} & & 1 \\
1 & : & 2
\end{array}\right]
$$

The $\operatorname{sum} s$ is equal to

$$
\left[\begin{array}{lll}
\overline{1} & 2 & \\
1 & & : T \\
\overline{2} & &
\end{array}\right]+\left[\begin{array}{lll}
\overline{1} & \overline{2} & \\
1 & & : T \\
2 & &
\end{array}\right]+\left[\begin{array}{lll}
\overline{1} & 3 & \\
1 & & : T \\
\overline{3} & &
\end{array}\right]+\left[\begin{array}{lll}
\overline{1} & \overline{3} & \\
1 & & : T \\
3 & &
\end{array}\right]+\left[\begin{array}{lll}
\overline{1} & 0 & \\
1 & & : T \\
0 & &
\end{array}\right] .
$$

It follows that on $O(7)$,

$$
\left[\begin{array}{lll}
\overline{1} & \overline{2} & \\
1 & & : T \\
2 & &
\end{array}\right]=-\left[\begin{array}{lll}
\overline{1} & 2 & \\
1 & & : T \\
\overline{2} & &
\end{array}\right]-\left[\begin{array}{lll}
\overline{1} & 3 & \\
1 & & : T \\
\overline{3} & &
\end{array}\right]-\left[\begin{array}{lll}
\overline{1} & \overline{3} & \\
1 & & : T \\
3 & &
\end{array}\right]-\left[\begin{array}{lll}
\overline{1} & 0 & \\
1 & : T \\
0 &
\end{array}\right]+\left[\begin{array}{lll}
\overline{1} & 1 \\
1 & 2
\end{array}\right] .
$$

We now deal with violations of condition (OS 3).

Lemma 5.3. Suppose that $S$ and $T$ are $\lambda$-tableaux with two columns. and that $S$ is not $O(n)$ standard, in that $\alpha_{j}+\beta_{j}=2 j$ for some $j$ with $\alpha_{j}=\beta_{j}$, and $S\left(\alpha_{j}, 1\right)=\bar{\jmath}, S\left(\alpha_{j}, 2\right)=j$ and $S\left(\alpha_{j}-1,2\right) \neq \bar{\jmath}$. Then $[S: T]$ can be expressed as one-half a signed sum of bideterminants of three types: (i) of shape $\mu$ where $|\mu|<|\lambda|$; (ii) of shape $\mu$ where $\mu \triangleleft \lambda$; (iii) of the form $[U: T]$ where $S \prec U$. The tableaux $U$ in (iii) and their signs in the signed sum are independent of T. The terms from (i) and (ii) are all 0 if $T=T^{\lambda}$.

Proof. Proceed as in the proof of the previous lemma. Since $S\left(\alpha_{j}, 1\right)=\bar{\jmath}$ and $j>\bar{\jmath}$, then $j$ does not occur in the first column of $S$; since $S\left(\alpha_{j}, 2\right)=j$ and $S\left(\alpha_{j}-1,2\right) \neq \bar{\jmath}$, then $\bar{\jmath}$ does not occur in the second column of $S$. So $j \in C$; in this case let $C^{\prime \prime}=C-\{j\}$. Argue as in the proof of the previous lemma, but now with $\mathcal{S}^{\prime \prime}$ given by replacing pairs $i, \bar{\imath}$ in $S$ by $i^{\prime}, \bar{\imath}^{\prime}$ where $i^{\prime} \in \mathcal{I}-C^{\prime \prime}$. This time, $\sum_{U \in \mathcal{S}^{\prime \prime}}[U: T]$ is equal to $[S: T]$ plus an exceptional term $\left[S^{*}: T\right]$ where $S^{*}$ comes from replacing $\bar{\jmath}, j$ in $S$ with $j, \bar{\jmath}$, plus a signed sum $s_{3}$ of bideterminants $[U: T]$ where $S \prec U$. From Lemma 4.2

$$
[S: T]+\left[S^{*}: T\right]+s_{3}=s_{1}
$$

where $s_{1}$ is a signed sum of bideterminants of shape $\mu$ where $|\mu|<|\lambda|$. The tableau $S^{*}$ is not $G L(n)$-standard; straightening $\left[S^{*}: T\right]$, from Lemma 3.2, gives

$$
\left[S^{*}: T\right]=[S: T]+s_{3}^{\prime}+s_{2}
$$

where $s_{3}^{\prime}$ is a signed sum of bideterminants $[V: T]$ of shape $\lambda$ where $V \succ S$, and $s_{2}$ is a signed sum of bideterminants of shape $\mu$ where $\mu \triangleleft \lambda$. Then

$$
2[S: T]+s_{3}+s_{3}^{\prime}+s_{2}=s_{1}, \quad 2[S: T]=s_{1}-s_{2}-\left(s_{3}+s_{3}^{\prime}\right),
$$

where $s_{1}$ satisfies (i) in the conclusion of the lemma, $s_{2}$ satisfies (ii), and $s_{3}+s_{3}^{\prime}$ satisfies (iii). 
A BASIS OF BIDETERMINANTS FOR THE COORDINATE RING OF THE ORTHOGONAL GROUP 17

Example. Consider the function on $O(6)$ given by

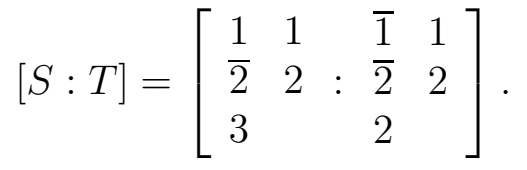

For the tableau $S, \alpha_{2}=\beta_{2}=2$. The 2 in $S$ is not protected, since $\overline{2}, 2$ occur in the second row, and there is no $\overline{2}$ above the 2 . From Lemma 4.2, on $O(6)$

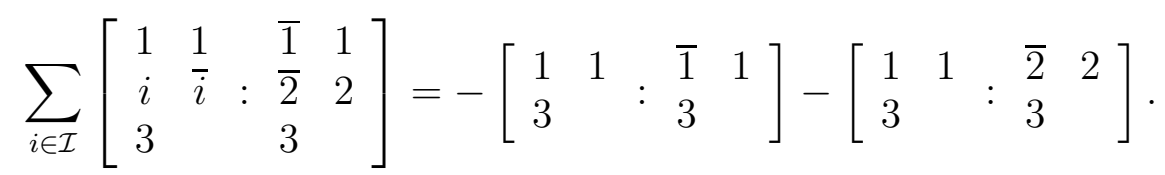

Let the right side of this equation be $s_{1}$. The left side of the equation is equal to

$$
\left[\begin{array}{lllll}
1 & 1 & & \overline{1} & 1 \\
\overline{2} & 2 & : & \overline{2} & 2 \\
3 & & & 3 &
\end{array}\right]+\left[\begin{array}{lllll}
1 & 1 & \overline{1} & 1 \\
2 & \overline{2} & : & \overline{2} & 2 \\
3 & & & 3 &
\end{array}\right]+\left[\begin{array}{lllll}
1 & 1 & & \overline{1} & 1 \\
\overline{3} & 3 & : & \overline{2} & 2 \\
3 & & & 3 &
\end{array}\right] .
$$

The first term is $[S: T]$. Call the third term $[V: T]$. The middle term needs to be $G L(n)$ straightened; this was done in Example 3.1. From this example, the middle term is equal to $[S: T]$, minus a bideterminant $[U: T]$, where $S \prec U$, plus a signed sum $s_{2}$ of bideterminants of shape $\left(2,1^{3}\right)$. So we have, on $O(6)$,

$$
2[S: T]=-[U: T]+[V: T]+s_{1}+s_{2}=s_{1}+s_{2}+s_{3} \text { where } s_{3}=-[U: T]+[V: T] .
$$

We can now state our main straightening result.

Theorem 5.1. Suppose that $S$ and $T$ are tableaux of the same shape $\lambda$, having at most $n$ parts. Then as functions on $O(n)$,

$$
[S: T]=\sum_{U} a_{U}[U: T]+s
$$

where the tableaux $U$ in the sum are $O(n)$-standard, each $a_{U} \in K$ and is independent of $T$, and $s$ is a linear combination of bideterminants of shapes $\mu$ where $|\mu|<|\lambda|$ or $|\mu|=|\lambda|$ and $\mu \triangleleft \lambda$. If $T=T^{\lambda}$ then $s=0$.

Proof. We use triple induction, first on the size $|\lambda|$ of a partition $\lambda$, next using the dominance order $\triangleleft$ on partitions of the same size, and lastly, downward induction on the order $\prec$ on tableaux of the same shape. Given a bideterminant $[S: T]$, use Mead's $G L(n)$-straightening from Theorem [3.1, to reach the case that $[S: T]$ is $G L(n)$-standard.

To achieve $O(n)$-standardness, we write the bideterminant $[S: T]$ as a product of two bideterminants, the first $[S: T]_{1}$ coming from the first two columns of each of $S$ and $T$, except for violations of (OS3) where we take columns 1 and $b$, and the second $[S: T]_{2}$ coming from the remaining columns. We need to straighten $[S: T]_{1}$. To get the condition that $\lambda_{1}^{\prime}+\lambda_{2}^{\prime} \leq n$, use Lemma 4.5. To achieve the three conditions in the definition of $O(n)$ standard, use the three previous lemmas. Then multiply by $[S: T]_{2}$. In the course of using these results, we sometimes replace a two-column subtableau with one where the columns have different lengths. We might get an array which is not a tableau, since the column lengths might not be decreasing. Then we rearrange the columns to get a tableau.

Using Remark 2.2, we have an analogous result where we replace $[S: T]$ by $[T: S]$. Then we have 
Corollary 5.1. The coordinate ring $K[O(n)]$ is spanned by $O(n)$-standard bideterminants.

\section{Filtrations and Linear Independence}

Let $K$ be a field of characteristic 0 .

The coordinate ring $K[G L(n)]$ is a $G L(n)$-bimodule,

$$
g_{1} f g_{2}(x)=f\left(g_{2} x g_{1}\right), \quad f \in K[G L(n)], g_{1}, g_{2}, x \in G L(n) .
$$

The same equation defines an $O(n)$-bimodule structure on the coordinate ring $K[O(n)]$.

Lemma 6.1. For tableaux $S$ and $T$ of the same shape $\lambda$, and $g \in G L(n)$,

$$
[S: T] g=\sum_{U} a_{U}[U: T]
$$

where the sum is over a certain collection $\{U\}$ of $\lambda$-tableaux, and each $a_{U} \in K$ does not depend on $T$.

Proof. For an $n \times n$ matrix $A$, let $[S: T](A)$ denote the evaluation of the function $[S: T]$ at $A$; in this notation, $([S: T] g)(X)=[S: T](g X)$.

First supppose that $S$ and $T$ each have just one column. From the Binet-Cauchy formula, (see for example [Pra, 2.3, p. 10]])

$$
[S: T](g X)=\sum_{U}[S: U](g)[U: T](X), \quad \text { so }[S: T] g=\sum_{U}([S: U](g))[U: T]
$$

where the $U$ in the sum vary over all column-increasing tableaux, of the same shape as $S$. In the general case, since a bideterminant is a product of determinants, one for each column of the tableaux, $[S: T]=\prod_{j}\left[S_{j}: T_{j}\right]$. Then

$$
\begin{aligned}
{[S: T] g } & =\prod_{j}\left[S_{j}: T_{j}\right] g=\prod_{j}\left(\sum_{U_{j}}\left(\left[S_{j}: U_{j}\right](g)\right)\left[U_{j}: T_{j}\right]\right) \\
& =\sum_{U}\left(\prod_{j}\left(\left[S_{j}: U_{j}\right](g)\right)\left[U_{j}: T_{j}\right]\right) \\
& =\sum_{U}\left(\prod_{j}\left[S_{j}: U_{j}\right](g)\right)\left(\prod_{j}\left[U_{j}: T_{j}\right]\right) \\
& =\sum_{U} a_{U}[U: T]
\end{aligned}
$$

where

$$
a_{U}=\prod_{j}\left[S_{j}: U_{j}\right] g=[S: U] g .
$$

For a partition $\lambda$ having at most $n$ parts, the Schur module for $G L(n)$, which we denote by $L^{\lambda}$, is the $K$-span of all bideterminants $\left[T^{\lambda}: T\right]$ where $T$ varies over all tableaux of shape $\lambda$. As is well known, this is a left $G L(n)$-submodule of $K[G L(n)]$; this also follows from Lemma 6.1. A good reference for Schur modules is Chapter 4 of $[\mathrm{G}]$, where they are denoted 
by $D_{\lambda, K}$. Since the characteristic of $K$ is $0, L^{\lambda}$ is irreducible. Define the right Schur module ${ }^{\lambda} L$ to be the $K$-span of all bideterminants $\left[T: T^{\lambda}\right]$.

Define the orthogonal left Schur module, denoted $L_{O}^{\lambda}$ to be the $K$-span of $\left[T^{\lambda}: T\right]_{O}$ as $T$ varies over all tableaux of shape $\lambda$. This is a left $O(n)$-submodule of $K[O(n)]$. Similarly we have the right orthogonal Schur module $\left[T: T^{\lambda}\right]_{O}$, spanned by $\left[T^{\lambda}: T\right]_{O}$.

Lemma 6.2. The right orthogonal Schur module ${ }^{\lambda} L_{O}$ is spanned over $K$ by all $O(n)$-standard bideterminants $\left[T: T^{\lambda}\right]$. The left analogue $L_{O}^{\lambda}$ is spanned by all $O(n)$-standard bideterminants $\left[T^{\lambda}: T\right]$.

Proof. The first statement follows from Theorem 5.1. The second statement follows by taking transposes.

We define the module $M^{\lambda}$, as in [KW, p. 254], as follows. Fix a partition $\lambda$, and let $T$ be a $\lambda$-tableau. Identify $T$ with an element of tensor space $V^{\otimes|\lambda|}$, and let $\{T\}=Y^{\lambda} T$ where $Y^{\lambda}$ is the Young symmetrizer, as in [KW, (2.2), p. 254]. Let $M^{\lambda}$ be the span of all $Y^{\lambda} T$, as $T$ varies over all $\lambda$-tableaux. This is a module for $G L(n)$, which as is well-known, is irreducible.

A trace tensor in $V^{\ell}$ is a linear combination of the form

$$
\sum_{i \in \mathcal{I}} x \otimes v_{i} \otimes y \otimes v_{\bar{\imath}} \otimes z
$$

where $x, y$ and $z$ are elements of some (possibly zero) tensor power of $V$ and $\left\{v_{i}: i \in \mathcal{I}\right\}$ is the standard basis of $V$. Let $U \subset V^{\otimes \ell}$ be the span of all such trace tensors. Define

$$
O^{\lambda}=M^{\lambda} /\left(M^{\lambda} \cap U\right) \text {. }
$$

As in $[\mathrm{KW}]$ and $\left[\mathrm{W}\right.$, Chap V.7], $O^{\lambda}$ is an irreducible $O(n)$-module, and the irreducible $O(n)$-modules are given by $O^{\lambda}$ where $\lambda$ is a partition with at most $n$ parts, with $\lambda_{1}^{\prime}+\lambda_{2}^{\prime} \leq n$.

In the $G L(n)$ case, it is well known that $M^{\lambda}$ and $L^{\lambda}$ are isomorphic. We will show that $O^{\lambda}$ and $L_{O}^{\lambda}$ are isomorphic $O(n)$-modules. To do this we use the following formalism. Let $M$ be a finite-dimensional polynomial $O(n)$-module, with basis $m_{1}, \ldots, m_{k}$. For $m \in M$, $g \in O(n)$, write

$$
g m=\sum_{i=1}^{k} a_{i}(g, m) m_{i} .
$$

It is clear that for $g, g^{\prime} \in O(n)$, we have $a_{i}\left(g g^{\prime}, m\right)=a_{i}\left(g, g^{\prime} m\right)$. Define $f_{m} \in K[O(n)]$ by $f_{m}(g)=a_{1}(g, m)$. (This, of course, depends on the choice of ordered basis of $M$.) Define the map

$$
F: M \rightarrow K[O(n)] \quad F(m)=f_{m}, \quad m \in M .
$$

Then $F$ is a homomorphism of $O(n)$-modules. We sometimes write $f_{M, m}$ instead of just $f_{m}$, and $F_{M}$ instead of $F$.

If we have another $O(n)$-module $M^{\prime}$ with basis $m_{1}^{\prime} \ldots, m_{\ell}^{\prime}$, we use the ordered basis $\left\{m_{i} \otimes m_{j}^{\prime}\right\}$ whose first element is $m_{1} \otimes m_{1}^{\prime}$. For the element $m \otimes m^{\prime}$, we have

$$
g\left(m \otimes m^{\prime}\right)=g m \otimes g m^{\prime}=\sum_{i, j} a_{i}(g, m) a^{\prime}\left(g, m^{\prime}\right) m_{i} \otimes m_{j}^{\prime}
$$

from which it follows that

$$
f_{M \otimes M^{\prime}, m \otimes m^{\prime}}=f_{M, m} f_{M^{\prime}, m^{\prime}} .
$$

If $N$ is an $O(n)$-sumbodule of $M$ which does not contain a scalar multiple of $m_{1}$, we pick the basis $m_{1}, \ldots, m_{k}$ of $M$ such that for some $j>1, m_{j+1}, \ldots, m_{k}$ is a basis of $N$. Let $\bar{M}$ 
denote $M / N$, which has basis $\bar{m}_{1}, \ldots, \bar{m}_{j}$. If $m \in M$ has the form $m=\sum_{i=1}^{k} a_{i} m_{i}$ then $\bar{m}=\sum_{i=1}^{j} a_{i} \bar{m}_{i}$ so $f_{\bar{M}, \bar{m}}=f_{M, m}$.

Suppose that $\lambda$ is the partition of $k$ whose shape consists of one column. It is well-known and easy to see that the module $G L(n)$-module $M^{\lambda}$ is the $k$-th exterior power $\bigwedge^{k} V$ of $V ; M^{\lambda}$ is an $O(n)$-module by restriction. Pick a basis of $M^{\lambda}$ whose first element is $m_{1}=v_{j_{1}} \wedge \cdots \wedge v_{j_{k}}$ where $j_{1}, j_{2}, \ldots j_{k}$ are the first $k$ elements of $\mathcal{I}$, as in (2.2). Let $T(k)$ denote the one-column tableau with entries $j_{1}, j_{2}, \ldots j_{k}$. Then $f_{M^{\lambda}, m_{1}}=[T(k): T(k)]_{O}$. Let $\wedge v(k)=v_{j_{1}} \wedge \cdots \wedge v_{j_{k}}$.

Now let $\lambda$ be any partition. Then $M^{\lambda}$ has a basis whose first element $m_{1}$ is $Y^{\lambda} T^{\lambda}$ which is equal to

$$
\wedge v\left(\lambda_{1}^{\prime}\right) \otimes \cdots \otimes \wedge v\left(\lambda_{\ell}^{\prime}\right) \in \bigwedge^{\left|\lambda_{1}^{\prime}\right|} V \otimes \cdots \otimes \bigwedge^{\left|\lambda_{\ell}^{\prime}\right|} V
$$

where $\lambda^{\prime}$ has $\ell$ parts. The module $M^{\lambda}$ is an $O(n)$-module by restriction. It follows that $f_{M^{\lambda}, m_{1}}$ is the bideterminant $\left[T^{\lambda}: T^{\lambda}\right]_{O}$.

Since $O^{\lambda}$ is a factor module of $M^{\lambda}$, it follows that $f_{O^{\lambda}, \bar{m}_{1}}=f_{M^{\lambda}, m_{1}}=\left[T^{\lambda}: T^{\lambda}\right]_{O}$. Since $O^{\lambda}$ is an irreducible $O(n)$-module, then any element $m \in O^{\lambda}$ is a linear combination of elements of the form $\left\{g_{i} \bar{m}_{1}\right\}$ for suitable $g_{i} \in O(n)$. Hence $F_{O^{\lambda}}(m) \in L_{O}^{\lambda}$. So $F_{O^{\lambda}}$ is an $O(n)$-module homomorphism from $O^{\lambda}$ to $L_{O}^{\lambda}$. From $[\mathrm{KW}], O^{\lambda}$ has basis given by standard $O(n)$-tableaux. Note that the main idea of $[\mathrm{KW}]$ is to prove straightening; linear independence follows from Proctor [Pro].

Proposition 6.1. For a partition $\lambda$ with at most $n$ parts, the left and right Schur modules $L_{O}^{\lambda}$ and ${ }^{\lambda} L_{O}$ are irreducible, and have $K$-bases given by $O(n)$-standard bideterminants $\left[T^{\lambda}: T\right]$, and $\left[T: T^{\lambda}\right]$, respectively.

Proof. From the preceding paragraph there is a non-zero homomorphism $F$ from $O^{\lambda}$ to $L_{O}^{\lambda}$. Since $O^{\lambda}$ has $K$-basis indexed by the set of $O(n)$-standard tableaux of shape $\lambda$, and since $L_{O}^{\lambda}$ is spanned by $O(n)$-standard $\left[T^{\lambda}: T\right]_{O}$ of shape $\lambda$, by Lemma 6.2 , it follows that $F$ is surjective. It is injective, since $O^{\lambda}$ is irreducible. The analogue for right Schur modules is similar.

Let $\leq$ be any partial order on the set of partitions of at most $n$ parts, such that

$$
\mu<\lambda \text { if }|\mu|<|\lambda| \text {; and } \mu<\lambda \text { if }|\mu|=|\lambda| \text { and } \mu \triangleleft \lambda \text {. }
$$

Let $A(\leq \lambda)$ denote the $K$-subspace of $K[O(n)]$ spanned by standard $O(n)$-bideterminants of shape $\mu$ where $\mu \leq \lambda$. Define $A(<\lambda)$ similarly. Let $\overline{A(\leq \lambda)}$ denote $A(\leq \lambda) / A(<\lambda)$. Let $\overline{[S: T]_{O}}$ denote the image of the bideterminant $[S: T]_{O}$ in $A(\leq \lambda) / A(<\lambda)$.

Theorem 6.1. There is an isomorphism

$$
\Phi: L_{O}^{\lambda} \otimes^{\lambda} L_{O} \rightarrow \overline{A(\leq \lambda))}
$$

of $O(n)$-bimodules given by

$$
\Phi\left(\left[T^{\lambda}: S\right]_{O} \otimes\left[T: T^{\lambda}\right]_{O}\right)=\overline{[T: S]_{O}}
$$

where $S$ and $T$ are $O(n)$-standard tableaux of shape $\lambda$.

Proof. Note that $\Phi$ is well-defined, since $L_{O}^{\lambda}$ and ${ }^{\lambda} L_{O}$ have bases, from Proposition 6.1. We first show that $\Phi$ is a right $O(n)$-module homomorphism. 
A BASIS OF BIDETERMINANTS FOR THE COORDINATE RING OF THE ORTHOGONAL GROUP 21

Suppose that $S$ and $T$ are $O(n)$-standard. For $g \in O(n)$, we have from Lemma 6.1

$$
\left[T: T^{\lambda}\right] g=\sum_{U} a_{U}\left[U: T^{\lambda}\right]
$$

where the $U$ in the sum are column-increasing tableaux, and $a_{U} \in K$. From Theorem 5.1, each $\left[U: T^{\lambda}\right]_{O}$ can be written as

$$
\left[U: T^{\lambda}\right]_{O}=\sum_{V} b_{U, V}\left[V: T^{\lambda}\right]_{O}
$$

where the tableaux $V$ are $O(n)$-standard. Then

$$
\begin{aligned}
\Phi\left(\left[T^{\lambda}: S\right]_{O} \otimes\left[T: T^{\lambda}\right]_{O} g\right) & =\Phi\left(\left[T^{\lambda}: S\right]_{O} \otimes \sum_{U, V} a_{U} b_{U, V}\left[V: T^{\lambda}\right]_{O}\right) \\
& =\sum_{U, V} a_{U} b_{U, V} \overline{[V: S]_{O}}
\end{aligned}
$$

On the other hand,

$$
\left(\Phi\left(\left[T^{\lambda}: S\right]_{O} \otimes\left[T: T^{\lambda}\right]_{O}\right)\right) g=\overline{[T: S]_{O} g} .
$$

From Lemma 6.1 the coefficients $a_{U}$ in (6.2) are the same for $[T: S] g$ as they are for $\left[T: T^{\lambda}\right] g$, so

$$
[T: S] g=\sum_{U} a_{U}[U: S]
$$

Moreover when $O(n)$-straightening $[U: S]_{O}$, we get the same answer, $\bmod A(<\lambda)$, as we do when straightening $\left[U: T^{\lambda}\right]_{O}$, from Theorem [5.1, So from (6.3) we get

$$
[U: S]_{O} \equiv \sum_{V} b_{U, V}[V: S]_{O} \bmod A(<\lambda)
$$

Then

$$
\left(\Phi\left(\left[T^{\lambda}: S\right]_{O} \otimes\left[T: T^{\lambda}\right]_{O}\right)\right) g=\overline{[T: S]_{O} g}=\sum_{U, V} a_{U} b_{U, V} \overline{[V: S]_{O}}
$$

It follows from this and (6.4) that $\Phi$ is a homomorphism of right $O(n)$-modules. That it is a homomorphism of left $O(n)$-modules has a similar proof.

Since $L_{O}^{\lambda}$ is an irreducible left $O(n)$-module and ${ }^{\lambda} L_{O}$ is an irreducible right $O(n)$-module, then $L_{O}^{\lambda} \otimes^{\lambda} L_{O}$ is an irreducible $O(n)$-bimodule. Therefore $\Phi$ has trivial kernel. Now $L_{O}^{\lambda} \otimes^{\lambda} L_{O}$ has $K$-basis given by the set of all $\left[T^{\lambda}: S\right] \otimes\left[T: T^{\lambda}\right]$ where $S$ and $T$ are $O(n)$-standard of shape $\lambda$, and this basis is mapped by $\Phi$ to the set $\left\{[T: S]_{O}\right\}$ of generators of $\overline{A(\leq \lambda)}$. It follows that $\Phi$ is an isomorphism.

Corollary 6.1. The set of $O(n)$-standard bideterminants $[S: T]_{O}$ are linearly independent.

Proof. This is because the set of all $\left[T^{\lambda}: S\right]_{O} \otimes\left[T: T^{\lambda}\right]$ is a basis of $L_{O}^{\lambda} \otimes{ }^{\lambda} L_{O}$.

Theorem 6.2. Let $<$ be a partial order satisfying (6.1), and let $\widetilde{<}$ be a total order which refines $<$. Then $A(\widetilde{\leq} \lambda)$ has $K$-basis given by the set of $O(n)$-standard bideterminants $[S: T]_{O}$ of shape $\mu \widetilde{\leq} \lambda$. 
Proof. By induction, $A(\widetilde{<} \lambda)$ has a basis given by $O(n)$-standard bideterminants of shape $\widetilde{<}$. It follows from Theorem 6.1 that the images of $[S: T]$ in $A(\leq \lambda) / A(<\lambda)$, where $[S: T]$ are $O(n)$-standard of shape $\lambda$, are a basis of $A(\leq \lambda) / A(<\lambda)$. We conclude that the $O(n)$-standard bideterminants of shape $\widetilde{\leq} \lambda$ form a basis of $A(\widetilde{\leq} \lambda)$.

Corollary 6.2. The set of $O(n)$-standard bideterminants is a $K$-basis of $K[O(n)]$.

Proof. From Theorem 5.1 the $O(n)$-standard bideterminants span $K[O(n)]$. Finitely many of them lie in $A(\tilde{\leq} \lambda)$ for some $\lambda$, hence are linearly independent.

\section{Base Change and Non-Zero Characteristic}

We first discuss base change. Suppose that $R$ is an integral domain of characteristic not 2 , having infinite field of fractions $K$. Define $O(n, R)$ to be the subgroup of $G L(n, R)$ given by elements $g$ such that $g^{t} J g=J$, where $J$ is the matrix of the bilinear form (2.1). Let $S O(n, R)$ be the elements in $O(n, R)$ of determinant 1. Essentially following [Bo1, 2.4] define $R[O(n)]$ to be the polynomial ring $R[X(i, j)]$ modulo the ideal $\mathcal{A}$ of polynomials which vanish on $O(n, \bar{K})$ where $\bar{K}$ is the algebraic closure of $K$. Define $R[S O(n)]$ similarly. From [Bo2, 18.3], $S O(n, K)$ is dense in $S O(n, \bar{K})$, in the Zariski topology, and then $O(n, K)$ is dense in $O(n, \bar{K})$. So $\mathcal{A}$ is the ideal of polynomials in $R[X(i, j)]$ which vanish on $O(n, K)$. In the case that $R$ is an infinite field $K$, the definition of $K[O(n)]$ just given agrees with the definition given in section 2, namely the restriction of polynomials in $K[X(i, j)]$ to $O(n, K)$.

The group $S O(n, K)$ is a Chevalley group. (Strictly speaking, for this to hold we should assume that $n \geq 4$.) We use a result of Chevalley, as discussed in [Bo1]. From [Bo1, Lemma 4.6] the ring $K \otimes_{\mathbb{Z}} \mathbb{Z}[S O(n)]$ is reduced (that is, it has no nilpotent elements). It follows, as in $[\mathrm{Bo} 1,3.4]$, that

$$
K[S O(n)] \cong K \otimes_{\mathbb{Z}} \mathbb{Z}[S O(n)]
$$

The group $O(n, K)$ has two irreducible components, namely the subsets on which the determinant is \pm 1 . We have the idempotents $e_{+}=(1 / 2)(1-\operatorname{det})$ and $e_{-}=(1 / 2)(1+$ det $)$ in $K[O(n)]$, and

$$
K[O(n)]=e_{+} K[O(n)] \oplus e_{-} K[O(n)], \quad e_{+} K[O(n)]=K[S O(n)] .
$$

Let $\mathbb{Z}[1 / 2]$ be the ring of rational numbers whose denominators are powers of 2 . It follows that

$$
K[O(n)] \cong K \otimes_{\mathbb{Z}[1 / 2]} \mathbb{Z}[1 / 2][O(n)] .
$$

Theorem 7.1. If $K$ is an infinite field of odd characteristic, then the set of $O(n)$-standard bideterminants $[S: T]$ is a $K$-basis of $K[O(n)]$.

Proof. We first prove an analogous result for $\mathbb{Z}[1 / 2][O(n)]$. Let $R=\mathbb{Z}[1 / 2]$.

Start with a polynomial $P$ in $R[X(i, j])$. Mead straightening can be done over any field, indeed over $\mathbb{Z}$. So $P$ is an $R$-linear combination of bideterminants $[S: T]$ where $S$ and $T$ are $G L(n)$-standard. A bideterminant $[S: T]$ is a polynomial in the $n^{2}$ variables $X(i, j)$ with integer coefficients, and so gives rise to an an element, denoted, $[S: T]_{O, R}$, of $R[O(n)]$. The $O(n)$-straightening results we have used, namely Lemmas 4.5, 5.1, 5.2, 5.3 hold in $R[O(n)]$. So we can do $O(n)$-straightening in $R[O(n)]$ and we see that the $O(n)$-standard bideterminants $[S: T]_{O, R}$ generate $R[O(n)]$ as an $R$-module. In $\mathbb{Q}[O(n)]$, the $O(n)$-standard bideterminants $[S: T]$ are linearly independent over $\mathbb{Q}$, from Theorem 6.2 , so the $O(n)$-standard 
bideterminants $[S: T]_{O, R}$ are linearly independent over $R$, from (7.2). Thus $R[O(n)]$ is a free $R$-module, with basis consisting of the $O(n)$-standard bideterminants $[S: T]_{O, R}$. Using (7.2), it follows that the $O(n)$-standard bideterminants $[S: T]$ form a $K$-basis of $K[O(n)]$.

We now consider Theorem 6.1 at odd positive characteristic. The definitions of $L_{O}^{\lambda},{ }^{\lambda} L_{O}$, and $\overline{A \leq \lambda}$ make sense for any field $K$. At positive characteristic, $L_{O}^{\lambda}$ is not in general an irreducible left $O(n, K)$-module, and we must find a different argument for the proof.

Let $R=\mathbb{Z}[1 / 2]$, and let $L_{O, R}^{\lambda}$ be the $R$-span of $\left[T^{\lambda}: T\right]_{O, R} \in R[O(n)]$ for all $\lambda$-tableaux $T$. This is a left $O(n, R)$ module, and has a basis of bideterminants $\left[T^{\lambda}: T\right]_{O, R}$ where $T$ is $O(n)$-standard of shape $\lambda$. There are analogous definitions and results for ${ }^{\lambda} L_{O, R}$.

As in the definition of $A(\lambda)$ following equation (6.1), let $A(\leq \lambda)_{R}$ be the $R$-span of all $[S: T]_{O, R}$ where $S$ and $T$ have shape $\mu \leq \lambda$. Similarly we define $A(<\lambda)_{R}$, and let $\overline{A(\leq \lambda)}_{R}=$ $A(\leq \lambda)_{R} / A(<\lambda)_{R}$. Then we have the following analogue of Theorem 6.1.

Theorem 7.2. If $K$ is an infinite field of characteristic not 2, then there is an isomorphism

$$
\Phi: L_{O}^{\lambda} \otimes^{\lambda} L_{O} \rightarrow{\overline{A(\leq \lambda))_{O}}}
$$

of $O(n, K)$-bimodules given by

$$
\Phi\left(\left[T^{\lambda}: S\right]_{O} \otimes\left[T: T^{\lambda}\right]_{O}\right)=\overline{[T: S]_{O}}
$$

where $S$ and $T$ are $O(n)$-standard tableaux of shape $\lambda$.

Proof. That $\Phi$ is a bimodule homomorphism is similar to the proof of Theorem 6.1. The isomorphism $\Phi$ above is defined over $R=\mathbb{Z}[1 / 2]$ :

$$
\Phi_{R}: L_{O, R}^{\lambda} \otimes{ }^{\lambda} L_{O, R} \rightarrow{\overline{A(\leq \lambda))_{O, R}}}, \quad \Phi_{R}\left(\left[T^{\lambda}: S\right]_{O, R} \otimes\left[T: T^{\lambda}\right]_{O, R}\right)=\overline{[T: S]_{O, R}}
$$

Since $\Phi$ is injective if $K=\mathbb{Q}$, then $\Phi_{R}$ is injective. It then follows that $\Phi_{R}$ is an isomorphism, and by base change we see that $\Phi$ is an isomorphism.

\section{The Group of orthogonal Similitudes}

Let $R$ be as in the previous section. With a view to future applications to the orthogonal Schur algebra, we now formulate our results with respect to the group $G O(n, R)$ of orthogonal similitudes. This is defined as all $g \in G L(n, R)$ such that for some unit $\gamma=\gamma(g)$ of $R$,

$$
\langle g v, g w\rangle=\gamma\langle v, w\rangle \text { for all } v, w \in R^{n} .
$$

Here $\langle$,$\rangle is the form defined in equation (2.1).$

The element $\gamma$ can be defined, as a function on $G O(n, R)$, by

$$
\gamma=\sum_{i \in \mathcal{I}} X(i, 1) X(\bar{\imath}, \overline{1})
$$

The map $\gamma: G O(n, R) \rightarrow R^{\times}$is a homomorphism.

Define $R[G O(n)]$ to be the polynomials in the $n^{2}+1$ variables $X(i, j)$ and $1 /$ det, with coefficients in $R$, modulo the ideal of those polynomials vanishing on $G O(n, K)$.

Lemma 8.1.

$$
K[G O(n)] \cong K \otimes_{\mathbb{Z}[1 / 2]} \mathbb{Z}[1 / 2][G O(n)]
$$


Proof. There are two cases, depending on whether $n$ is even or odd, since $G O(n)$ is connected if $n$ is odd but not if $n$ is even. For $g \in G O(n, K)$ the condition (8.1) is equivalent to $g^{t} J g=\gamma J$, where $J$ is the matrix of the bilinear form (2.1); then on $G O(n, K)$,

$$
\operatorname{det} g^{2}=\gamma(g)^{n} \text {. }
$$

If $n$ is odd, then from [KMRT, 12.4], $G O(n, K)=S O(n, K) \cdot K^{\times} \cong S O(n, K) \times K^{\times}$. It follows from the definitions that

$$
\mathbb{Z}[G O(n)] \cong \mathbb{Z}[S O(n)] \otimes \mathbb{Z}\left[t, t^{-1}\right]
$$

where $t$ is an indeterminate. Then the lemma follows from (7.1).

Now suppose that $n$ is even. Given $c \in K^{\times}$let $\xi(c)$ be the diagonal matrix

$$
\operatorname{diag}(c, c, \ldots, c, 1,1, \ldots 1)
$$

where the first $n / 2$ diagonal entries are equal to $c$. We have a map of algebraic varieties

$$
\Phi: O(n) \times K^{\times} \rightarrow G O(n), \quad \Phi(g, c)=g \xi(c) .
$$

For $g \in G O(n, K)$,

$$
\gamma\left(g \xi\left(\gamma(g)^{-1}\right)=\gamma(g) \gamma(g)^{-1}=1, \quad \text { so } \quad g \xi\left(\gamma(g)^{-1}\right) \in O(n) .\right.
$$

Then $\Phi$ is invertible, with inverse given by

$$
\Psi(g)=\left(g \xi(\gamma(g))^{-1}, \gamma(g)\right)
$$

and $\Psi$ is a regular map because of (8.2). It follows that

$$
K[G O(n)] \cong K[O(n)] \otimes K\left[t, t^{-1}\right] .
$$

and the lemma follows from (7.2).

Define $A(n, r, R)$ to be the $R$-module of polynomials of degree $r$ in the $n^{2}$, variables $X(i, j)$, with coefficients in $R$. Define $A_{G O}(n, r, R)$ to be $A(n, r, R)$ modulo the submodule of all polynomials which vanish on $G O(n, K)$, so $A_{G O}(n, r, K)$ is the restriction of the polynomials in $A(n, r, K)$ to $G O(n, K)$. We will find a basis for $A_{G O}(n, r, K)$.

The main technical Lemma 4.1, becomes, in this context:

Lemma 8.2. As functions on $G O(n, K)$, we have

$$
\sum_{\substack{i_{1}, \ldots, i_{a} \\
\in \mathcal{I}-C}}\left[\begin{array}{ccc}
i_{1} & \bar{\imath}_{1} \\
i_{2} & \bar{\imath}_{2} \\
\vdots & \vdots \\
i_{a} & \bar{\imath}_{a} \\
S_{0}
\end{array}\right]=a ! \sum_{d=1}^{a} \gamma^{d} \mathcal{S}_{d}
$$

Proof. Proceed as with the proof of Lemma 4.1, until equation (4.3). As functions on $O(n, K), \sum_{i \in \mathcal{I}} X(i, j) X(\bar{\imath}, k)=\delta_{j, \bar{k}}$, whereas as functions on $G O(n, K)$, we have

$$
\sum_{i \in \mathcal{I}} X(i, j) X(\bar{\imath}, k)=\delta_{j, \bar{k}} \gamma
$$

Then equation (4.3) gets replaced, as functions on $G O(n, R)$, by

$$
\sum_{i_{s} \in \mathcal{I}-C} X\left(i_{s}, \sigma\left(g_{s}\right)\right) X\left(\bar{\imath}_{s}, \tau\left(h_{s}\right)\right)=\delta_{\sigma\left(g_{s}\right), \overline{\tau\left(h_{s}\right)}} \gamma-\sum_{i_{s} \in C} X\left(i_{s}, \sigma\left(g_{s}\right)\right) X\left(\bar{\imath}_{s}, \tau\left(h_{s}\right)\right) .
$$


Similarly, all occurences of $\delta_{\sigma\left(g_{s}\right), \overline{\tau\left(h_{s}\right)}}$ get replaced by $\delta_{\sigma\left(g_{s}\right), \overline{\tau\left(h_{s}\right)}} \gamma$, and $\delta(s)$ gets replaced by $\delta_{\sigma\left(g_{s}\right), \overline{\tau\left(h_{s}\right)}} \gamma$. Equation (4.4) becomes, on $G O(n, K)$,

$$
\prod_{s=1}^{a}\left(\delta(s)-\sum_{i_{s} \in C} Z\left(s, i_{s}\right)\right)=\sum_{D \in \mathcal{D}}(-1)^{\ell(D)} \gamma^{d} \sum_{\left(i_{1}, \ldots, i_{\ell}\right) \in C^{\ell}} \prod_{s \in D^{\prime}} Z\left(s, i_{s}\right) .
$$

With these replacements, the result follows as in the proof of Lemma 4.1.

Our main result now, for any infinite field $K$ of characteristic not 2, is

Theorem 8.1. A basis of $A_{G O}(n, r, K)$ is given by the set of functions on $G O(n, K)$

$$
\mathcal{B}_{r}=\left\{\gamma^{k}[S: T]: k \in \mathbb{Z}, \quad 0 \leq k \leq r / 2\right\}
$$

where $[S: T]$ is $O(n)$-standard of shape $\lambda$ with $|\lambda|=r-2 k$.

Proof. As in the previous section, we first find a basis for $A_{G O}(n, r, \mathbb{Z}[1 / 2])$. We start with $G L(n)$-straightening, which can be done over any ring $R$. We need to show that as functions on $G O(n, Q)$, a bideterminant $[S: T]$ where $S$ and $T$ are tableaux of shape $\lambda$ with $|\lambda|=r$, is a $\mathbb{Z}[1 / 2]$-linear combination of the elements in $\mathcal{B}_{r}$. We call this $G O(n)$-straightening. We need $G O(n)$-analogues of our $O(n)$-straightening lemmas. From Lemma 8.2 we get a $G O$ analogue of Lemma 4.2, which is that on $G O(n, \mathbb{Q})$

$$
\sum_{U \in \mathcal{S}^{*}}[U: T]=\sum_{d=1}^{a} \gamma^{d} \mathcal{S}_{d}
$$

As in Lemma 5.1, $[S: T]=\sum_{U \in \mathcal{S}}-[U: T]+s$; using (8.4), we see that on $G O(n, Q) s$ is a signed sum of terms of the form $\gamma^{b}\left[S^{\prime}: T^{\prime}\right]$ where $S^{\prime}$ is a tableaux of shape $\sigma$ where $|\sigma|<|\lambda|$ and $b+|\sigma|=r$. Since $\left[S^{\prime}: T^{\prime}\right]$ can be $G O(n)$-straightened by induction, then so can $[S: T]$. Analogues of Lemmas 5.2 and 5.3 are similar.

We need a $G O$-analogue of Lemma 4.5. Equation (4.9) becomes, on $G O(n, \mathbb{Q})$,

$$
[S: T]= \pm \operatorname{det}^{2} \gamma^{\ell} \cdot\left[\bar{S}_{1}^{\prime}: \bar{T}_{1}^{\prime}\right]\left[\bar{S}_{2}^{\prime}: \bar{T}_{2}^{\prime}\right]
$$

for a suitable integer $\ell$. If $[S: T]$ has shape $\lambda$ where $\lambda_{1}^{\prime}+\lambda_{2}^{\prime}>n$, the analogue of Lemma 4.5, using (8.3), is that on $G O(n, \mathbb{Q}),[S: T]= \pm \gamma^{b}[\widetilde{S}: \widetilde{T}]$, for a suitable integer $b$, where $\widetilde{S}$ and $\widetilde{T}$ are as in Lemma 4.5. This proves $G O(n)$-straightening.

Since a polynomial in $\mathbb{Z}[1 / 2][X(i, j)]$ which vanishes on $G O(n, \mathbb{Q})$ also vanishes on $O(n, \mathbb{Q})$, we have a ring homomorphism

$$
F: A_{G O}(n, r, Z[1 / 2]) \rightarrow \mathbb{Z}[1 / 2][O(n)] .
$$

The set $F\left(\mathcal{B}_{r}\right)$ is linearly independent, since $\gamma=1$ on $O(n, \mathbb{Q})$ and the $O(n)$-standard bideterminants are linearly independent in $\mathbb{Q}[O(n)]$. So $\mathcal{B}_{r}$ is a linearly independent set in $A_{G O}(n, r, Z[1 / 2])$. The theorem now follows from Lemma 8.1 .

\section{REFERENCES}

[B] A. Berele, Construction of Sp-modules by tableaux, Linear and Multilinear Algebra 19 (1986), 299307.

[Bo1] A. Borel, Properties and linear representations of Chevalley groups, in "Seminar on Algebraic Groups and Related Finite Groups (The Institute for Advanced Study, Princeton, N.J., 1968/69)" pp. 1-55, Lecture Notes in Mathematics 131, Springer, Berlin/Heidelberg/New York, 1970.

[Bo2] A. Borel, Linear Algebraic Groups, 2nd ed, Springer, New York, 1991. 
[dC] C. de Concini, Characteristic free "decomposition" of the coordinate ring of the symplectic group, in "Non-Commutative Structures in Algebra and Geometric Combinatorics" (A. de Luca, ed.), pp. 121-128, Quaderni della ricerca scientifica 109, Rome, 1981.

[DEP] C. de Concini, D. Eisenbud, C. Procesi, Young diagrams and determinantal varieties, Invent. Math. 56 (1980), 129-165.

[Don] S. Donkin, Representations of symplectic groups and the symplectic tableaux of R. C. King, Linear and Multilinear Algebra 29 (1991), 113-124.

[Dot] S. Doty, Polynomial representations, algebraic monoids, and Schur algebras of classical type, J. Pure Appl. Algebra 123 (1998), 165-199.

[DRS] P. Doubilet, G.-C. Rota, J. Stein, On the foundations of combinatorial theory. IX. Combinatorial methods in invariant theory, Studies in Appl. Math. 53 (1974), 185-216.

[G] J. A. Green, Polynomial Representations of $G L_{n}$, Lecture Notes in Mathematics 830, Springer, Berlin/Heidelberg/New York, 1980.

[KW] R. C. King, T. A. Welsh, Construction of orthogonal group modules using tableaux. Linear and Multilinear Algebra 33 (1993), 251-283.

[KMRT] M.-A. Knus, A. Merkurjev, M. Rost, J.-P. Tignol, The Book of Involutions, American Mathematical Society, Providence, 1998.

[K] M. Koppinen, Good bimodule filtrations for coordinate rings, J. London Math. Soc. (2) 30 (1984), 244-250.

[L] G. Lusztig, Introduction to Quantum Groups, Birkhäuser, Boston, 1993.

$[\mathrm{M}] \quad$ D. G. Mead, Determinantal ideals, identities, and the Wronskian, Pacific J. Math. 42 (1972), 165-175.

[O] S. Oehms, Centralizer coalgebras, FRT-construction, and symplectic monoids, J. Algebra 244 (2001), $19-44$.

[Pra] V. V. Prasolov, Problems and Theorems in Linear Algebra, Translations of Mathematical Monographs. 134 (American Mathematical Society, Providence, RI, 1994).

[Pro] R. A. Proctor, Young tableaux, Gel'fand patterns, and branching rules for classical groups, J. Algebra 164 (1994), 299-360.

[W] H. Weyl, The Classical Groups, Princeton University Press, Princeton, N.J., 1946.

University of Alberta, Department of Mathematical and Statistical Sciences, Edmonton, Alberta, Canada T6G 2G1

E-mail address: gcliff@math.ualberta.ca 